\title{
Ultrafast electron diffraction: Excited state structures and chemistries of aromatic carbonyls
}

\author{
Sang Tae Park, Jonathan S. Feenstra, and Ahmed H. Zewail ${ }^{\text {a) }}$ \\ Laboratory for Molecular Science, and Physical Biology Center for Ultrafast Science and Technology, \\ California Institute of Technology, Pasadena, California 91125
}

(Received 23 February 2006; accepted 14 March 2006; published online 4 May 2006)

\begin{abstract}
The photophysics and photochemistry of molecules with complex electronic structures, such as aromatic carbonyls, involve dark structures of radiationless processes. With ultrafast electron diffraction (UED) of isolated molecular beams it is possible to determine these transient structures, and in this contribution we examine the nature of structural dynamics in two systems, benzaldehyde and acetophenone. Both molecules are seen to undergo a bifurcation upon excitation $\left(S_{2}\right)$. Following femtosecond conversion to $S_{1}$, the bifurcation leads to the formation of molecular dissociation products, benzene and carbon monoxide for benzaldehyde, and benzoyl and methyl radicals for acetophenone, as well as intersystem crossing to the triplet state in both cases. The structure of the triplet state was determined to be "quinoidlike" of $\pi \pi^{*}$ character with the excitation being localized in the phenyl ring. For the chemical channels, the product structures were also determined. The difference in photochemistry between the two species is discussed with respect to the change in large amplitude motion caused by the added methyl group in acetophenone. This discussion is also expanded to compare these results with the prototypical aliphatic carbonyl compounds, acetaldehyde and acetone. From these studies of structural dynamics, experimental and theoretical, we provide a landscape picture for, and the structures involved in, the radiationless pathways which determine the fate of molecules following excitation. For completeness, the UED methodology and the theoretical framework for structure determination are described in this full account of an earlier communication [J. S. Feenstra et al., J. Chem. Phys. 123, 221104 (2005)]. (c) 2006 American Institute of Physics.
\end{abstract}

[DOI: $10.1063 / 1.2194017]$

\section{INTRODUCTION}

The aromatic carbonyl molecules, exemplified by benzaldehyde and acetophenone, have been of experimental interest for decades. These molecules with close-lying $n \pi^{*}$ and $\pi \pi^{*}$ excited states in both singlet and triplet manifolds are ideally suited for the study of the physical processes of intersystem crossing and phosphorescence, as well as photochemical reactions of valence isomerization, bond breakage, and atom transfer. In two recent reports, ${ }^{1,2}$ we have presented our preliminary ultrafast electron diffraction results on the photophysical and photochemical channels of benzaldehyde and acetophenone. In this paper, we determine the relevant structures, time scales, and the bifurcation of pathways. We also give a full account of the experimental and theoretical methodologies, and discuss the results and their significance toward the understanding of numerous spectroscopic observations.

A feature shared by both benzaldehyde and acetophenone is a complex manifold of excited states with simultaneous and competitive photophysical and photochemical processes. Ambiguities abound in the pathways, products, and time scales. The mechanisms by which $S_{2}$ excited benzaldehyde yields molecular dissociation products while ex-

\footnotetext{
${ }^{a)}$ Author to whom correspondence should be addressed. Electronic mail: zewail@caltech.edu
}

cited acetophenone homolytically cleaves are important issues that portend major advances in understanding excited state decay processes. In order to address the nature of these processes, dark structures of excited state radiationless transitions and chemistries must be determined on the ultrashort time scale.

This paper is outlined as follows: Section II highlights previous spectroscopic findings. In Secs. III and IV the experimental and theoretical frameworks are discussed, respectively. In Sec. V we present the diffraction theory needed and the methodology used for structure determination. In Sec. VI the results of electron diffraction studies on both molecules in their ground states and following $266.7 \mathrm{~nm}$ femtosecond excitation to their $S_{2}$ states are described, and in Sec. VII we discuss the significance of these findings in the context of the global landscape for bifurcation into photophysical and photochemical processes. First, however, we shall present salient features of the photophysics and photochemistry of the aromatic carbonyls studied here (see Fig. 1).

\section{RADIATIVE AND NONRADIATIVE PROCESSES}

\section{A. Benzaldehyde}

The absorption spectrum of vapor-phase benzaldehyde with wavelengths longer than $260 \mathrm{~nm}$ shows two broad peaks. The lower peak is highly structured and corresponds 
Benzaldehyde

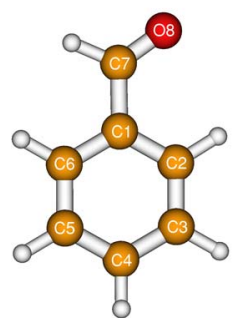

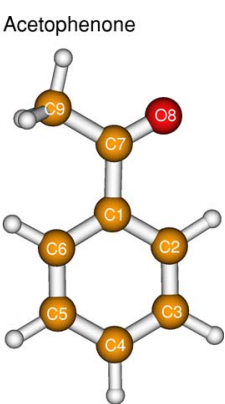

FIG. 1. Molecular structures of benzaldehyde and acetophenone.

to a transition to the $S_{1}\left(n \pi^{*}\right)$ state. The higher-energy peak, marking transition to the $S_{2}\left(\pi \pi^{*}\right)$ state, is much more intense and shows only a weak structure. ${ }^{3}$ Absorption into either of these states, and the $S_{3}\left(\pi \pi^{*}\right)$ that lies above, results in phosphorescence. ${ }^{4,5}$ This particular feature of benzaldehyde has been exploited by numerous studies attempting an explanation of its photophysics. Experiments to this end have been performed on benzaldehyde in the vapor, ${ }^{3-20}$ in solution, $^{21}$ and in matrices. ${ }^{22-24}$

The proximity and nature of low-lying $n \pi^{*}$ and $\pi \pi^{*}$ states of both singlet and triplet manifolds have been a main focus both experimentally and theoretically. The origin of $S_{1}$ $\left(n \pi^{*}\right)$ was found to lie $26919 \mathrm{~cm}^{-1}$ above the ground state by sensitized phosphorescence ${ }^{8}$ and $26920 \mathrm{~cm}^{-1}$ above by sensitized surface electron ejection. ${ }^{19}$ It is slightly blueshifted in hexane solution $\left(26960 \mathrm{~cm}^{-1}\right)$ (Ref. 21) and in p-dibromobenzene matrices $\left(27284 \mathrm{~cm}^{-1}\right){ }^{25} S_{2}\left(\pi \pi^{*}\right)$ has its origin at 35200 and $35191 \mathrm{~cm}^{-1}$ relative to the ground state as seen in the phosphorescence excitation spectrum ${ }^{11}$ and ionization excitation spectrum, ${ }^{13}$ respectively. Another $\pi \pi^{*}$ state, $S_{3}$, has its $0-0$ transition at $41334 \mathrm{~cm}^{-1}$, as noted by UV absorption. ${ }^{26}$ In the triplet manifold, matters are more complex since the $n \pi^{*}$ and $\pi \pi^{*}$ states are very close lying. Emission in the vapor is detected only from the $n \pi^{*}\left(T_{1}\right)$; however, in certain matrix environments the $\pi \pi^{*}$ may be shifted lower in energy allowing direct spectral observation. The $T_{1}$ origin in the vapor phase is readily distinguished by phosphorescence and is assigned between 25180 and $25190 \mathrm{~cm}^{-1}{ }^{8,9,17-19}$ Similar to the values for the $S_{1}$ origin, values for $T_{1}$ in liquid hexane ${ }^{21}$ and in a $p$-dibromobenzene matrix $^{25}$ are blueshifted to 25195 and $25515 \mathrm{~cm}^{-1}$, respectively. Some band congestion in the vapor phosphorescence spectrum $\sim 1000 \mathrm{~cm}^{-1}$ above the $T_{1}$ origin is cautiously assigned to intensity borrowed by the $T_{2}\left(\pi \pi^{*}\right)$ state. $8,9,17,19$ The caution in this assignment arises due to the theoretical suggestion that the spacing between the levels is, in fact, large enough not to cause a significant breakdown of the Born-Oppenheimer approximation. ${ }^{12,18,27}$ Theory places the $T_{1}-T_{2}$ energy gap between 726 and $4190 \mathrm{~cm}^{-1}$ depending on the method of calculation. ${ }^{28-31}$ In matrices, however, the ${ }^{3} n \pi^{*}$ and ${ }^{3} \pi \pi^{*}$ levels switch and both are observed; the $\pi \pi^{*}$ state is lower by $162-\sim 2000 \mathrm{~cm}^{-1}$ and varies highly with the choice of host. ${ }^{23,25}$

Decay of the excited state population by phosphorescence is the most widely known relaxation pathway in gasphase benzaldehyde and the quantum yield of emission ap- proaches unity when excitation is near the $S_{1}$ origin. ${ }^{15}$ It continues to be observed, albeit with less intensity, as excitation reaches the $S_{3}$ state. Two conflicting reports of the phosphorescence behavior at higher excitation energies exist in the literature. In several accounts it is reported that dropoffs in yield occur near both the $S_{2}$ and $S_{3}$ thresholds. $5,7,12,16$ Yet in another account, the drop-offs are refuted as gas-cell "wall effects" and simply a monotonic decrease is reported. ${ }^{4}$ Independent of the exact behavior, a nonradiative channel depleting the excited state population becomes important above $S_{1}$ excitation making phosphorescence less significant. An intermediate state invoked in the nonradiative decay route lives long enough for collision-induced deactivation as added gas causes an increase in phosphorescence yield. ${ }^{7,11,12,14,16}$ The phosphorescent state itself is found to live in the range of milliseconds to nanoseconds, shortening with increased excess energy. ${ }^{4,10,14,19}$ Lifetime information is also available for the $S_{2}$ state by femtosecond photoelectron spectroscopy. It was observed to live for $440 \mathrm{fs}$ at the origin and undergoes a monotonic lifetime decrease with increasing excitation energy, leaving the state by ultrafast internal conversion. $^{20}$

The structures of the electronic states of benzaldehyde are largely obtained through theoretical means ${ }^{29-31}$ with some experimental exceptions. The ground state has been studied by both microwave spectroscopy ${ }^{32}$ and electron diffraction $^{33}$ in the gas phase, and the barrier to formyl group torsion is found to be 1715 and $>1700 \mathrm{~cm}^{-1}$, respectively. Additionally, analysis of the phosphorescence spectra and $S_{1}$ excitation spectra shows the $\mathrm{C}=\mathrm{O}$ stretch as the most intense band with prominent progression, confirming the $n \pi^{*}$ nature of both states as well as their structural similarity to the ground state (except for the $\mathrm{C}=\mathrm{O}$ length). ${ }^{8}$

In addition to the rich photophysics studied in this molecule, there exist chemical pathways resulting in radical and molecular dissociation products. The first thorough experiments on the photochemistry of benzaldehyde ${ }^{11}$ show dissociation into benzene and carbon monoxide upon photolysis by $276 \mathrm{~nm}$ radiation ( $S_{2}$ excitation). The products were extracted from the cell and identified by gas chromatography. Being relatively insensitive to added oxygen, the molecular dissociation products are determined to have formed through a "concerted" reaction and not through radical intermediates. At low pressures the quantum yield of benzene is found to be 0.89 , and the addition of foreign gases causes the benzene yield to decrease while increasing the yield of phosphorescence. A model is proposed with short and long benzene formation channels; the long channel may be deactivated to the phosphorescent state $\left(T_{1}\right)$ by collisions. ${ }^{11}$ An insensitivity to collisions with added $\mathrm{O}_{2}$ further supports the nonradical nature of the reaction, as the yield of benzene was unchanged. ${ }^{6}$ The nature of the reaction was conclusively verified in experiments where benzaldehyde- $d_{6}$ was mixed with the benzaldehyde- $h_{6}$ and photolyzed with $276 \mathrm{~nm}$ radiation. ${ }^{12}$ Only the concerted intramolecular reaction products benzene- $\mathrm{d}_{6}$ and benzene- $\mathrm{h}_{6}$ were identified. Yield of the dissociation products was near unity. ${ }^{12}$ Benzene ion has also been detected in mass spectrometry experiments pumping at $248 \mathrm{~nm}^{34}$ 
Although ultrafast dynamics was hitherto lacking, some time scales of benzene formation have been obtained. In an ionization experiment using single shots of $2 \mathrm{~ns}$ pulses, ${ }^{35}$ benzene ions were detected with $258.9 \mathrm{~nm}$ excitation but not with $258.7 \mathrm{~nm}$. For both excitations benzene is formed within the pulse, but because ground state benzene has a resonant absorption at $258.9 \mathrm{~nm}$, but not at $258.7 \mathrm{~nm}$, it is efficiently ionized and observed at $258.9 \mathrm{~nm}$. Furthermore, the photoelectron spectrum was recorded at both excitation energies for benzaldehyde as well as benzene. No photoelectrons were detected at $258.7 \mathrm{~nm}$, but the spectrum observed using $258.9 \mathrm{~nm}$ matched that of ground state benzene. In addition to confirmation of the product, these experiments put an upper bound of $2 \mathrm{~ns}$ on the photoreaction. ${ }^{35}$ In another report, single shots at $266 \mathrm{~nm}$ showed no benzene ion signal when the pulse width was $25 \mathrm{ps,} \mathrm{but} \mathrm{showed} \mathrm{a} \mathrm{near-unity}$ production of benzene ions when the excitation laser pulse width was $8 \mathrm{~ns} .^{36,37}$

Using $10 \mathrm{~ns}$ pulses at $266 \mathrm{~nm}$ in a mass spectrometer Polevoi et al. determined the time constant for benzene ion formation to be $\sim 30 \mathrm{~ns}$. Their kinetic model predicts that the reaction corresponds to triplet benzaldehyde dissociating into benzene in its own triplet state and ground state carbon monoxide. ${ }^{38}$ The most recent work on benzaldehyde photochemistry by Silva and Reilly, a time-resolved mass spectrometry study using $3-5$ ns pulses, contributes valuable additional information. ${ }^{39}$ With excitation into $S_{2}$ and time delayed photoionization by the $157 \mathrm{~nm}$ probe laser, both benzene and parent benzaldehyde ions are detected. The ratio of benzene to benzaldehyde ions increases with a decreasing excitation wavelength - complete dissociation occurring near $260 \mathrm{~nm}$ excitation. Two decay times are observed for the benzaldehyde ion: a short decay of $<1 \mu$ s and a longer decay of $>1 \mu \mathrm{s}$. The shorter decay time nearly coincides with the time constant of benzene ion appearance and is $\sim 80 \mathrm{~ns}$ at $270 \mathrm{~nm}$ excitation. Since the probe pulse $(157 \mathrm{~nm})$ is only intended to ionize electronically excited benzenes, the benzene ion signal is believed to be from the triplet. However, ground state benzene is also identified in their results by its photoionization excitation spectrum and must be formed via a different pathway. The threshold for benzene formation is placed somewhere near (but below) the $S_{2}$ origin. ${ }^{39}$

Radical products were reported only once from $S_{2}$, after gas phase photolysis of benzaldehyde by $7 \mathrm{~ns}$ pulses of 280 , 285 , and $308 \mathrm{~nm}$ radiations. HCO radicals were detected by absorption and quantum yields were estimated at $0.3-0.4 .^{40}$ Based on the evidence in numerous previous works, the radicals must surely be products of a multiphoton reaction. The chemistry observed upon excitation into the $S_{2}$ state contrasts with the different chemistries observed through $S_{1}$. Several researchers report no chemistry at all after $S_{1}$ excitation. ${ }^{4,12,14,36,37}$ Chemistry that is reported is of a fragmentation nature resulting in the benzoyl radical and atomic hydrogen. ${ }^{22,41}$ One study notes a slow buildup of polymer on the walls of the gas cell after prolonged exposure at 328 or $365 \mathrm{~nm} .{ }^{11}$ Hydrogen atoms were also produced in the thermal breakdown of benzaldehyde above $1000 \mathrm{~K}$. CO may also be lost from the resulting fragment above $1150 \mathrm{~K} .{ }^{42}$

\section{B. Acetophenone}

The states of acetophenone are similarly ordered as benzaldehyde, with two close-lying triplet states (the lower one being strongly phosphorescent) located just below the $S_{1}$ origin. There are many experimental measurements and theoretical estimates of the relative energies of the states in the gas phase. $S_{1}\left(n \pi^{*}\right)$ is found to lie $27279 \mathrm{~cm}^{-1}$ above $S_{0}$ by its sensitized phosphorescence. ${ }^{8}$ The origin of the $S_{2}\left(\pi \pi^{*}\right)$ excitation spectrum is $35402 \mathrm{~cm}^{-1}$ above $S_{0}$ (Ref. 43) and the $0-0$ by optoacoustic spectroscopy is reported at $34010 \mathrm{~cm}^{-1}$. ${ }^{6}$ The $S_{3}\left(\pi \pi^{*}\right)$ origin is seen at $41695 \mathrm{~cm}^{-1}$ by absorption in a jet. ${ }^{26}$ In the triplet manifold, the well-known $T_{1}\left(n \pi^{*}\right)$ state has its origin at $25791 \mathrm{~cm}^{-1}$ by sensitized phosphorescence ${ }^{8}$ and $25786 \mathrm{~cm}^{-1}$ by its direct emission. ${ }^{44}$ Like benzaldehyde, the $T_{2}\left(\pi \pi^{*}\right)$ state was not directly observed in the vapor. A congestion of spectral features noted $600 \mathrm{~cm}^{-1}$ above the $T_{1}$ origin has been attributed to possible mixing with the nearby $T_{2}{ }^{8}$ In general, the vapor-phase spectrum is weaker than that of benzaldehyde, with a less prominent progression of the carbonyl stretching bands-perhaps from a greater contribution of $\pi \pi^{*}$ character. ${ }^{9}$ Unlike benzaldehyde, emission from only a single phosphorescent site is observed in certain matrices and believed to correspond to some highly perturbed state. Indeed, the spectrum shows features from both states precluding it from being unequivocally assigned to either the ${ }^{3} n \pi^{*}$ or ${ }^{3} \pi \pi^{*}$. The conclusion is that the $T_{1}-T_{2}$ energy gap is even less than that of benzaldehyde. ${ }^{23,44}$ The energies and geometries of these lowlying electronic states have been studied theoretically, yielding similar results. An estimate of the $T_{1}-T_{2}$ energy gap is only $565 \mathrm{~cm}^{-1}$. 30,31

Like for benzaldehyde, the prolific study of acetophenone is largely due to its intense phosphorescence facilitated by efficient intersystem crossing from $S_{1}$ into the triplet manifold. The photophysics of acetophenone have been studied in the gas phase, ${ }^{6-9,20,26,43-47}$ solution, ${ }^{21,48}$ and in matrices. ${ }^{22,23,44}$ Phosphorescence detected in the vapor phase is seen to decrease in intensity with increasing photon energy and exhibits a drop-off as excitation approaches $S_{2}$. The phosphorescence quantum yield at the $S_{1}$ origin is near unity, ${ }^{7,45,47}$ while at the $S_{2}$ origin it is reduced to $\sim 10^{-4} .{ }^{43}$ The drop-off is attributed to the rise of an intermediate state that can be collisionally deactivated-increased phosphorescence yield is observed with the addition of foreign gas. ${ }^{7,45,47}$ A kinetic model predicts a lifetime of $380 \mathrm{~ns}$ for the intermediate. ${ }^{7}$ Again, like benzaldehyde, the presence of a drop-off is contested with contrary evidence depicting a monotonic decrease. ${ }^{47}$

Although phosphorescence lifetimes are known to be in the microsecond to nanosecond range, the lifetimes of other states have gone largely unmeasured. By linewidth measurements the lifetime of the $S_{2}$ origin is found to be $260 \mathrm{fs}$, ${ }^{43}$ and a subsequent femtosecond time-resolved photoelectron experiment puts it at $140 \mathrm{fs}$, faster than that of benzaldehyde. The faster decay is likely the consequence of more efficient radiationless relaxation mediated by extra accepting modes from the methyl group. ${ }^{20}$ The lifetime of the Rydberg state reached by $193 \mathrm{~nm}$ absorption is instrument limited and 
UED setup: Top view

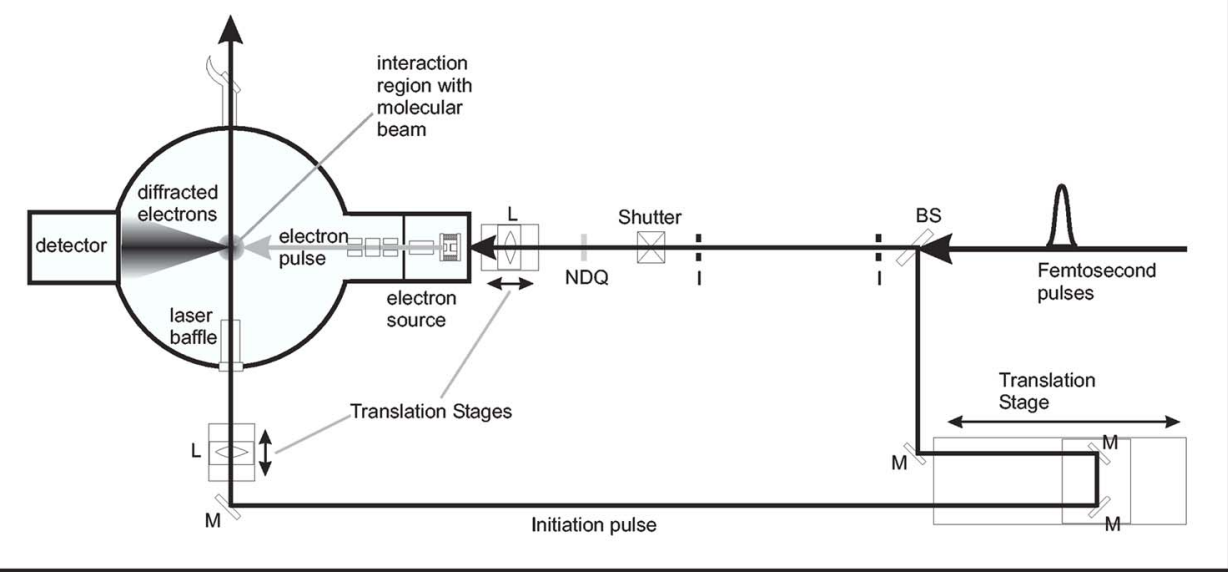

FIG. 2. Schematic diagram of the UED setup. The output of the amplified femtosecond laser system is split such that a majority of the power is time delayed and used to initiate the reaction in the molecular beam. The weak light pulses produce electron pulses which intersect the pump laser and molecular beam in the diffraction chamber. Diffracted electrons generate signal on a digital detector.
$<200$ fs. $^{49}$ These fast lifetimes and the experimentally known (and theoretically predicted) ${ }^{30}$ efficiency of intersystem crossing are consistent with the lack of fluorescence from acetophenone. Fluorescence has been observed, however, from acetophenone derivatives in solution with attached aromatic groups. ${ }^{50}$

Experimental information regarding the structures of the excited states is confined to the $S_{1}$ and $T_{1}$ states. The carbonyl stretching progression observed is indicative of $n \pi^{*}$ states with the main structural difference from the ground state being a lengthening of the $\mathrm{C}=\mathrm{O}$ bond. ${ }^{8}$ Experimental information on the $\pi \pi^{*}$ structures is limited to acetophenone derivatives where the ${ }^{3} \pi \pi^{*}$ state is lower in energy. Substituted acetophenones were excited in solution with $266 \mathrm{~nm}$ light after which (500 ns) infrared spectra were recorded. The changes in the spectrum indicate a structural difference with the ground state mainly in the aromatic ring consistent with the quinoid structure of a $\pi \pi^{*}$ state. $^{51}$ Theoretical inquiries back up these experimental results reaffirming structural differences with the ground state localized in the $\mathrm{C}=\mathrm{O}$ bond for $n \pi^{*}$ states and in the aromatic ring for $\pi \pi^{*}$ states. $^{30,31}$ It is to be noted that no experimentally determined structure exists for ground state acetophenone.

Acetophenone photochemistry has attracted less interest than that of benzaldehyde. Unlike the molecular dissociation seen in the aldehyde, radical products represent the photochemistry of this aromatic ketone. Carbon monoxide was not detected in the earliest of experiments by Berger and Steel. ${ }^{45}$ Instead, the major products are the benzoyl and methyl radicals observed at multiple excitations ranging from 282 to $193 \mathrm{~nm}$ and also seen as products in strong-field dissociation experiments. The other radical cleavage products, phenyl and acetyl radicals, are also observed but with a much lower yield. ${ }^{45,52,53}$ Like benzaldehyde, the yield of chemical products is seen to approach unity with $S_{2}$ excitation at the lowest pressures but decreases coincident with increasing phosphorescence when foreign gas is added, suggesting a vibrationally excited intermediate to fragmentation. Only trace amounts of the molecular dissociation products of carbon monoxide and toluene were detected after photolysis by
193 and $248 \mathrm{~nm}$ radiations; a branching ratio with the radical products of $\sim 1: 100 .^{53}$ Little to no decomposition products are noted upon $S_{1}(365 \mathrm{~nm})$ excitation. $^{45}$

\section{EXPERIMENT}

The third-generation ultrafast electron diffraction apparatus at Caltech has been briefly presented elsewhere, ${ }^{54,55}$ and here we provide the detailed methodology that makes these experiments possible. The ultrafast electron diffraction (UED) experimental apparatus is a combination of several components that will be addressed separately: a femtosecond laser system, a high-vacuum chamber, a high-voltage ultrafast pulsed electron gun, a charge-coupled device (CCD) detector, and a high-temperature inlet system. Simply summarized, the beam from the femtosecond laser system is split and the more intense pulses are focused and directed into a molecular beam to initiate reaction. The weaker femtosecond laser pulses are focused onto a back-illuminated silver photocathode in the electron gun to generate electron pulses by the photoelectric effect. The pulses are accelerated and focused to meet the excitation laser and the molecular beam in a mutually perpendicular arrangement. Scattered electrons are detected by a CCD camera at the terminus of a phosphor, fiber-optic, image-intensifier chain. The excitation laser pulses may be delayed to provide time-dependent diffraction patterns. A schematic drawing of the setup is shown in Fig. 2.

Samples used in these experiments were obtained from Aldrich: acetophenone (99\%), benzaldehyde $(99.5+\%)$, and 1,3-butadiene $(>99 \%)$, and Air Liquide: carbon dioxide $(99.5 \%)$ and xenon $(99.995 \%)$. All samples were used without further purification.

\section{A. Femtosecond laser system}

The laser system used in these UED experiments is a mode locked Ti:sapphire oscillator (Tsunami) which delivers pulses (6 nJ; $800 \mathrm{~nm} ; 60 \mathrm{fs} ; 80 \mathrm{MHz}$ ) to a dual-stage Ti:sapphire amplifier (Super Spitfire). Amplified pulses $(2.3 \mathrm{~mJ}$, $\sim 120 \mathrm{fs}, 800 \mathrm{~nm} ; 1 \mathrm{kHz})$ are frequency tripled in a thirdharmonic generator module (Uniwave) to provide ultraviolet 


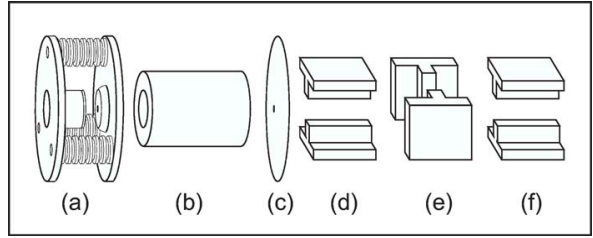

FIG. 3. Elements of the pulsed electron source. Femtosecond laser pulses enter the electron source from the left. The photocathode and anode (a) are separated by $3 \mathrm{~mm}$ and experience a potential of $30 \mathrm{kV}$. Electrons pass through a hole in the anode and are focused by the magnetic lens (b). The electron source is separated from the scattering chamber by a $2 \mathrm{~mm}$ aperture (c). Vertical (d) and horizontal (e) deflection plates and vertical streaking plates (f) are used to adjust the electron beam position and to measure its temporal width.

pulses $(400 \mu \mathrm{J} ; 266.7 \mathrm{~nm})$. The UV beam then enters the "optics table" (see Fig. 2) where it is split into electrongeneration and excitation pulses. The electron-generation beam ( $\sim 7 \%$ of the original beam) passes through two irises closed to $\sim 1 \mathrm{~mm}$ to attenuate the beam and improve its transverse mode. The beam also passes through an NDQ100 (10\% transmission) neutral density filter for further attenuation. A lens with a focal length of $300 \mathrm{~mm}$ then focuses the beam into the electron gun. The significant attenuation of the electron-generation beam is necessary to prevent damage to the photocathode which must maintain its integrity for the duration of the experiment. A computer controlled, modified camera shutter prevents electron extraction except during data acquisition.

The excitation beam (93\% of the original beam from the tripler) is time delayed by a translation stage before it, too, is focused by a movable lens into the diffraction chamber. The translation stage has a range of approximately $1.5 \mathrm{~ns}$; a laser pulse can arrive in the scattering chamber $\sim 1 \mathrm{~ns}$ before an electron pulse.

\section{B. Ultrafast electron system}

The pulsed electron gun (Fig. 3) is housed in a chamber separated from the diffraction chamber by a $2 \mathrm{~mm}$ aperture. The chamber is pumped by a BOC Edwards turbomolecular pump (EXT 250; 200 1/s) by which pressure is maintained at $\sim 10^{-7}$ torr during experiments (nearly independent of molecular beam operation). Before entering the chamber, laser pulses pass through two irises (each at $\sim 1 \mathrm{~mm}$ ) and a NDQ100 (10\% transmittance) to attenuate the beam such that the photocathode is not damaged over the course of the experiment. Laser pulses are focused with an adjustable lens and enter the chamber to impinge on a back-illuminated photocathode. The photocathode consists of a stainless steel mount to which a sapphire window is fastened by silver paste. A silver coating of $25 \mathrm{~nm}$ is deposited on the window using a BOC Edwards Auto 306 vacuum coater.

A voltage of $30 \mathrm{kV}$ is maintained via high-voltage feedthrough and a high-voltage power supply and a grounded extraction anode is positioned $3 \mathrm{~mm}$ from the coated surface by ridged Macor spacers. The electron beam passes through a $200 \mu \mathrm{m}$ pinhole on the extraction anode to clean the profile. Accelerated electrons have $\lambda_{\text {de Broglie }}$ $=0.06979 \AA$ [see below, Sec. V B, Eq. (5)] and are traveling at $9.8 \times 10^{7} \mathrm{~m} / \mathrm{s}$ (nearly $1 / 3$ the speed of light). Leaving the extraction region, electrons are focused with an electromagnetic coil by which the size and shape are optimized $[\sim 400 \mu \mathrm{m}$ full width at half maximum (FWHM) with Gaussian profile]. The electrons then pass through the $2 \mathrm{~mm}$ pinhole separating the electron gun chamber from the scattering chamber. Once in the scattering chamber, two sets of deflection plates (horizontal and vertical) and a set of streak plates (vertical) are used to manipulate the electron beam's path and its final position on the detector. The streak plates are used in the streaking experiments that measure the temporal profile of the electron pulses. In addition, they are also needed to move the beam outside of the filtered region on the detector (see below) where the number of electrons present in a single pulse may be measured accurately.

Calibrations have been performed relating the temporal pulse width to the number of electrons per pulse so that streak measurements do not have to be performed prior to every experiment. ${ }^{54}$ The pulse properties for these experiments were measured to be $8 \times 10^{4}$ electrons/pulse for acetophenone and $3 \times 10^{4}$ electrons/pulse for benzaldehyde. The FWHMs of the electron beams were measured to be $\sim 370$ and $\sim 360 \mu \mathrm{m}$ for benzaldehyde and acetophenone, respectively, and the pulsewidths in these experiments were typically less than 6 or 20 ps depending on the number of electrons per pulse.

The photocathode must undergo a period of "warming up" where the number of electrons increases over time with exposure of the photocathode to the laser. This typically takes $12 \mathrm{~h}$, after which the beam is stable and ready for diffraction experiments.

\section{Diffraction chamber and molecular beam}

The scattering chamber is separated from its primary pumping system (Varian VHS-6 oil diffusion pump, $2400 \mathrm{l} / \mathrm{s}$, with a liquid nitrogen baffle) by a pneumatic gate valve. The pump permits vacuum to $10^{-7}$ torr when the molecular beam is not running. Pressures during the experiment can be as high as $3 \times 10^{-4}$ torr. Electron scattering from background gas is minimized by the use of a liquid nitrogen cryotrap which is a baffle (Varian) attached to the diffusion pump.

Stray excitation laser light in the scattering chamber is minimized by a baffle that extends approximately $10 \mathrm{~cm}$ from the $\mathrm{CaF}_{2}$ inlet window. After many hours of experimentation with certain organics, background gas can lead to the formation of a layer of polymeric coating on the inlet window and this reduces the laser transmission. After crossing the molecular and electron beams in the interaction region, excitation laser light exits the chamber through a quartz window at Brewster's angle. The top view schematic of the scattering chamber is shown in Fig. 2.

The inlet manifold was rebuilt prior to the experiments on benzaldehyde and acetophenone in order to accommodate their high boiling points. The current inlet is constructed entirely of stainless steel. A sample reservoir is capable of holding $300 \mathrm{ml}$ of sample and the valves can tolerate heats of up to $400{ }^{\circ} \mathrm{C}$. A manifold is connected to the high-temperature 
portion of the inlet through a fine-metering needle valve. The manifold allows $\mathrm{CO}_{2}, \mathrm{Xe}$, and butadiene access to the nozzle. The manifold is also connected to a mechanical pump for evacuation during sample changes.

The nozzle tip, with a $180 \mu \mathrm{m}$ aperture, is connected to the manifold via a stem running through an insulated flange. The nozzle (and stem) is heated by a tight wrap of an insulated high-resistance thermocouple wire, the manifold is wrapped in a fiberglass heating tape, and the reservoir is clamped with a custom band heater (Watlow). The heating systems of each unit are monitored and controlled separately. The temperatures are optimized to prevent condensation clogging and thermal decomposition while still delivering an appropriate amount of sample to the interaction region. Vaporized sample from the reservoir passes through the manifold, stem, and nozzle to form a molecular beam via effusive expansion. For benzaldehyde the temperatures of the nozzle, manifold, and sample were 210,230 , and $129^{\circ} \mathrm{C}$, respectively. The temperature of the sample reservoir was increased to $148{ }^{\circ} \mathrm{C}$ over the course of the experiment in order to keep constant the pressure and the dependent scattering signal. For acetophenone the temperatures of the nozzle, manifold, and sample were 230,260 , and $110^{\circ} \mathrm{C}$, respectively, and the sample temperature was increased to $120^{\circ} \mathrm{C}$ during this much shorter experiment.

The size of the molecular beam is measured by admitting gas $\left(\mathrm{CO}_{2}\right)$ to the chamber and moving the nozzle across the stationary electron beam. The scattering intensity at each nozzle position is plotted and fitted with a Lorentzian curve. The width of the electron beam is deconvoluted to yield the molecular beam width. The nozzle must be $800-900 \mu \mathrm{m}$ above the electron beam for a molecular beam width similar in size to those of the laser and electron beams. The molecular beam sizes for the benzaldehyde and acetophenone experiments were measured to be 380 and $270 \mu \mathrm{m}$, respectively.

\section{Clocking and zero of time}

Optimizing the overlap between the electron, laser, and molecular beams in the scattering chamber and determining the temporal relationship between electron and laser pulses are done simultaneously. Rough overlap optimization of the molecular beam with electron beam and laser beam is aided by the shadow cast by the nozzle and viewed on the CCD and through the outlet window, respectively. Overlap is finetuned by observing and maximizing a photoion-induced lensing effect on the electron beam. ${ }^{56}$ Gas (1,3-butadiene) is delivered to the scattering chamber via the inlet nozzle and the laser and electron beams are admitted to intersect with it. The undiffracted electron beam profile is monitored while the time delay of the laser pulses is changed. The laser causes ionization of the gas and the subsequent charge separation alters the shape of the transmitted electron beam. The time at which the electron beam shape begins to change from circular to elliptical give the in situ time zero. ${ }^{56}$ The position of the laser is fine-tuned using the inlet mirror and focusing lens to maximize the lensing effect.

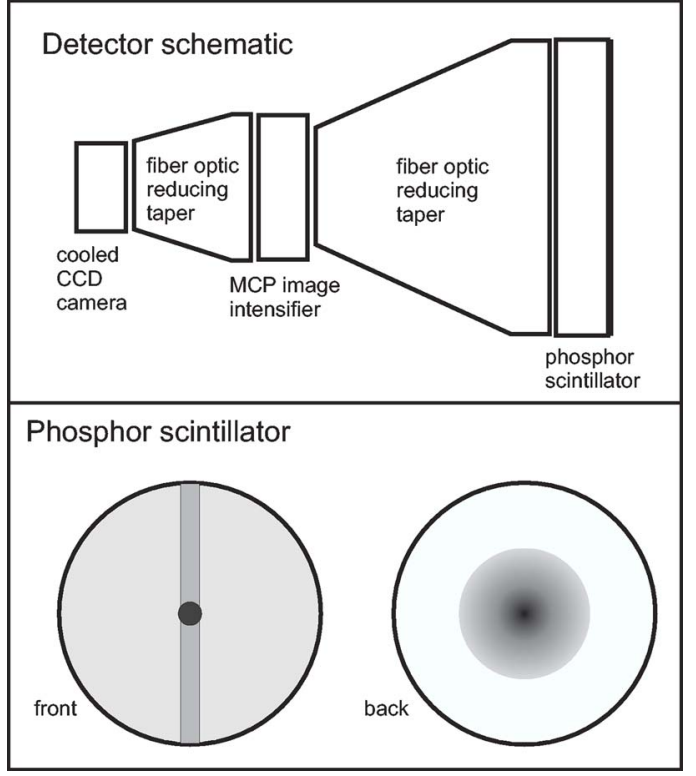

FIG. 4. (top) Side view of the detector schematic. (bottom) Front and back views of the phosphor screen showing the vertical null stripe and central beam stop and the phosphor-coated face plate with the radial symmetric filter.

The overall experimental time resolution is governed by the laser pulse width, the electron pulse width, and the group velocity mismatch. Using the above-mentioned beams' properties and published formulas, ${ }^{54,57}$ the overall time resolutions of the benzaldehyde and acetophenone experiments were derived.

\section{E. Detector and data acquisition}

Detection of the electron diffraction signal is done with a CCD camera at the end of an image-intensifier fiber-optic chain (see Fig. 4). The phosphor scintillator is comprised of a fiber-optic disk (diameter $=8 \mathrm{~cm}$ ) with a coating of P-47 phosphor. A $300 \mathrm{~nm}$ layer of aluminum is applied to block any scattered UV light. A radial symmetric neutral-density filter is coated on the reverse side of the fiber-optic disk in order to increase the dynamic range of detection (the electron diffraction signal decreases rapidly with a scattering angle). Electrons strike the phosphor screen and emit photons which then travel through the disk and the fiber-optic reducing taper to the image intensifier (Hamamatsu) which amplifies the signal via a microchannel plate. The output photons traverse a second fiber-optic reducing taper and are detected by a CCD camera (Photometrics, KAF-1000). The CCD camera is cooled to $-40{ }^{\circ} \mathrm{C}$ in order to reduce noise. The entire setup is capable of single electron detection $(\sim 100$ detector intensity units/electron). The CCD camera consists of a 1024 $\times 1024$ pixel array. The effective pixel size is $57.8 \mu \mathrm{m}$ on the side of the phosphor screen, and pixels are binned $2 \times 2$ during the experiments.

Since the vast majority $(\sim 99 \%)$ of the electron beam passes through the molecular beam unscattered, a small metal cup mounted on an aluminum strip covers the center of the phosphor scintillator to prevent saturation of the detector. In addition, the image-intensifier itself has a null stripe bi- 
secting along the vertical, separating the left and right halves which can be operated separately. Exposure of the CCD and data acquisition is entirely computer controlled. The standard exposure time is 240000 pulses (4 min). Multiple exposures (typically 100) are acquired and averaged in the data processing (see below).

One hundred diffraction patterns (4 min exposures at $1 \mathrm{kHz}$ ) are taken for each of background electron scattering (no molecular beam), carbon dioxide, xenon, ground state (molecular sample without excitation laser), each timeresolved benzaldehyde data point $(-100,-50,-10,-5,+0$, $+5,+10,+15,+20,+30,+40,+50,+100$, and $+1000 \mathrm{ps})$, and each time-resolved acetophenone data point $(-100,+50$, and $+100 \mathrm{ps})$. Conversion of the pattern data to the scattering intensity curves used in the data analysis will be dealt with in detail in Sec. V.

\section{STRUCTURE QUANTUM CALCULATIONS}

Structural refinement in UED requires an initial guess geometry. Although one could, in principle, use a chemically intuitive guess, our data analysis is always begun with the structural results of $a b$ initio or density functional theory (DFT) calculations. Ideally, regardless of the choice of initial guess, structural refinement should converge to one single true structure. In practice, however, this is not always true, especially when there are a large number of structural parameters involved and the fitting surface contains many local minima. Therefore, it is a good practice to have an initial guess as close to the true structure as possible. For this, a high level of theoretical calculation is desired, such as DFT, coupled cluster (CC), quadratic configuration interaction with single and double excitations (QCISD), or multiconfiguration self-consistent field (MCSCF). In our study, all calculations have been carried out using either GAUSSIAN98 (Ref. 58) or GAMESS (Ref. 59) packages. The details of data analysis and structural refinement in UED are covered in Sec. V.

\section{A. Ground state}

For the structures of molecules in the singlet $\left(S_{0}\right)$ or triplet $\left(T_{1}\right)$ ground states, highly accurate results are available at fairly low computational costs. The B3LYP level ${ }^{60}$ with the $6-311 \mathrm{G}(d, p)$ basis set has been found useful in determining structure, energy, and vibrational frequencies. $^{54,61}$

Geometry optimization for the ground state of reactants and possible products of benzaldehyde and acetophenone systems were carried out at the B3LYP level using the 6$311 \mathrm{G}(d, p)$ basis set. UB3LYP/6-311G $(d, p)$ was used for radical product candidates such as those involving $\mathrm{H}$, methyl, formyl, acetyl, benzoyl, and phenyl radicals as well as for the triplet state of molecules. Planar symmetry was imposed for the singlet ground states of benzaldehyde and acetophenone and the $T_{1}$ state of benzaldehyde, and frequency calculations were performed to ensure minimum energy configurations. For the $T_{1}$ state of acetophenone, the symmetry constraint was lifted, since the methyl orientation deviates from planar symmetry; the skeleton re- mains nearly planar. The theoretically determined relative energies of the parent and product molecules are listed in Table I and compared to experimental values where available; the agreement is satisfactory. Experimental values of the changes in heats of formation at $298 \mathrm{~K}$ were taken from NIST (Ref. 62) and then converted to $0 \mathrm{~K}$ values using the theoretical frequencies obtained from DFT calculations.

\section{B. Excited state}

Several methods are available for calculating the properties of excited states. Among them, MCSCF uses a minimum set of orbitals (active space) for a configuration interaction (CI) calculation; just enough to determine the relevant excited state characteristics. ${ }^{63}$

MCSCF calculations were performed for singlet and triplet, ground and excited states, of benzaldehyde and acetophenone to explore structural changes during the photophysical processes. An active space was chosen to account for the carbonyl $\pi^{*} \leftarrow n$ and phenyl $\pi^{*} \leftarrow \pi$ transitions in both molecules. Specifically, ten electrons were partitioned into nine orbitals: one oxygen nonbonding orbital, one each of $\mathrm{C}=\mathrm{O} \pi$ and $\pi^{*}$ orbitals, and three each of $\mathrm{C}=\mathrm{C} \pi$ and $\pi^{*}$ orbitals. The $6-31 \mathrm{G}(d)$ basis set was used to reproduce previous work on benzaldehyde by Wang et al. ${ }^{31}$ Following this confirmation, calculations were then conducted using the 6-311G $(d, p)$ basis set. The initial molecular orbitals were guessed from the B3LYP calculations with virtual orbital optimizations after SCF convergence. Using the guessed orbitals, which were properly reordered to form nine active spaces, vertical energies were calculated and molecular orbital characters were visually checked to confirm the corresponding transitions observed in the experiments. Full structural optimizations were followed by vibration frequency calculations to check the effect of imposing planar symmetry. Multireference perturbation corrections (MCQDPT) (Ref. 64) were applied to evaluate the electron correlation energies. Relative energies of excited states are listed in Table II and compared with experimental values where available.

In calculating reactive pathways from the $n \pi^{*} S_{1}$ excited state of benzaldehyde, the SCF suffered convergence failure; the selected initial guess wave functions do not remain effective when large nuclear coordinate changes are implemented. ${ }^{65}$ For unknown reasons, preparing the initial guess vector at the rearranged geometry was also unsuccessful. Since the $S_{1}$ MCSCF reaction pathway calculations failed, UB3LYP/6-311G $(d, p)$ was employed to study the reaction on the $n \pi^{*} T_{1}$ surface instead. Both the $S_{1}{ }^{1} n \pi^{*}$ and $T_{1}{ }^{3} n \pi^{*}$ states of acetophenone and benzaldehyde have nearly identical stationary geometries and vibrational frequencies. The similarities permit results found for $T_{1}$ benzaldehyde to be carefully adapted to the $S_{1}$ surface; a correction of $3906 \mathrm{~cm}^{-1}$ is used, the difference between the experimental $S_{1}$ origin and the DFT $T_{1}$ origin. Later discussion of the $S_{1}$ dynamics is made under the assumption that calculations on $T_{1}$ provide information relevant to its singlet counterpart. 
TABLE I. Theoretical and experimental energetics of benzaldehyde and acetophenone: chemistries.

\begin{tabular}{|c|c|c|c|c|}
\hline \multirow[b]{2}{*}{ Molecule } & \multirow[b]{2}{*}{ Channel } & \multicolumn{2}{|c|}{ Energy $\left(\mathrm{cm}^{-1}\right)$} & \multirow[b]{2}{*}{ Temperature $^{\mathrm{c}}(\mathrm{K})$} \\
\hline & & Theor. $^{a}$ & Expt. $^{\text {b }}$ & \\
\hline \multirow[t]{7}{*}{ Benzaldehyde } & $\mathrm{C}_{6} \mathrm{H}_{5} \mathrm{CHO}\left(S_{0}^{*}\right)$ & 0 & 0 & 2379 \\
\hline & $\mathrm{C}_{6} \mathrm{H}_{5} \mathrm{CHO}\left(T_{1}\right)$ & 23015 & 25183 & 1307 \\
\hline & $\mathrm{C}_{6} \mathrm{H}_{5} \mathrm{CHO}\left(T_{2}\right)$ & 25148 & $25183 \sim 26183$ & 1161 \\
\hline & $\mathrm{C}_{6} \mathrm{H}_{6}\left(S_{0}\right)+\mathrm{CO}$ & 840 & 460 & 2696 \\
\hline & $\mathrm{C}_{6} \mathrm{H}_{6}\left(T_{1}\right)+\mathrm{CO}$ & 30489 & $30087^{\mathrm{d}}$ & 1044 \\
\hline & $\mathrm{H}+\mathrm{C}_{6} \mathrm{H}_{5} \mathrm{CO}$ & 30403 & $\mathrm{n} / \mathrm{a}^{\mathrm{e}}$ & 954 \\
\hline & $\mathrm{C}_{6} \mathrm{H}_{5}+\mathrm{HCO}$ & 32335 & 34637 & 993 \\
\hline \multirow[t]{7}{*}{ Acetophenone } & $\mathrm{C}_{6} \mathrm{H}_{5} \mathrm{COCH}_{3}\left(S_{0}^{*}\right)$ & 0 & 0 & 2058 \\
\hline & $\mathrm{C}_{6} \mathrm{H}_{5} \mathrm{COCH}_{3}\left(T_{1}\right)$ & 23569 & 25791 & 1157 \\
\hline & $\mathrm{C}_{6} \mathrm{H}_{5} \mathrm{COCH}_{3}\left(T_{2}\right)$ & 25643 & $\mathrm{n} / \mathrm{a}^{\mathrm{e}}$ & 1068 \\
\hline & $\mathrm{C}_{6} \mathrm{H}_{5} \mathrm{CH}_{3}\left(S_{0}\right)+\mathrm{CO}$ & 1860 & 1814 & 2227 \\
\hline & $\mathrm{C}_{6} \mathrm{H}_{5} \mathrm{CH}_{3}\left(T_{1}\right)+\mathrm{CO}$ & 30662 & $30890^{\mathrm{f}}$ & 945 \\
\hline & $\mathrm{CH}_{3}+\mathrm{C}_{6} \mathrm{H}_{5} \mathrm{CO}$ & 26660 & $\mathrm{n} / \mathrm{a}^{\mathrm{e}}$ & 1284 \\
\hline & $\mathrm{C}_{6} \mathrm{H}_{5}+\mathrm{CH}_{3} \mathrm{CO}$ & 31200 & 34266 & 972 \\
\hline
\end{tabular}

${ }^{\mathrm{a}}$ Theoretical energies were evaluated from B3LYP/6-311G $(d, p)$ level calculations, except for the energies of the $T_{2}$ states of benzaldehyde and acetophenone which were evaluated using $\operatorname{MCSCF}(10,9) / 6-311 \mathrm{G}(d, p)$.

${ }^{\mathrm{b}}$ Experimental values were evaluated from the change in the heat of formation at $298 \mathrm{~K}$ (Ref. 62) and converted to the $0 \mathrm{~K}$ value using DFT frequencies.

${ }^{\mathrm{c}}$ Temperatures for dissociation products are upper limits where all the excess energies are partitioned into vibrational energy; none for relative translational energy and rotational energy.

${ }^{\mathrm{d}}$ The energy of $\mathrm{C}_{6} \mathrm{H}_{6}\left({ }^{3} B_{1 u}\right)$ is $29627 \pm 12 \mathrm{~cm}^{-1}$ by mass-analyzed threshold ionization (MATI) of discharged benzene (Ref. 79). The energy of $\mathrm{C}_{6} \mathrm{H}_{6}\left({ }^{3} B_{1 u}\right)$ is $3.665 \mathrm{eV}$ from electron-energy-loss (EEL) data (Ref. 80).

${ }^{\mathrm{e}}$ These data were not available.

${ }^{\mathrm{f}}$ The energy of $\mathrm{C}_{6} \mathrm{H}_{5} \mathrm{CH}_{3}\left(T_{1}\right)$ is $3.605 \mathrm{eV}(0-0)$ for the solid (Ref. 81) and $3.8 \mathrm{eV}$ (maximum) for the gas (Ref. 82).

\section{DIFFRACTION THEORY AND METHODOLOGY}

\section{A. 2D diffraction patterns and the detector function}

As mentioned in Sec. III E, a spatial filter on the back side of the phosphor scintillator increases the dynamic range of detection by leveling the rapid drop-off of scattering intensity. However, this effect must be accounted for in order to obtain the molecular scattering function. The experimental raw patterns, $F^{e}$, containing the filter effect, $\eta(x, y)$, can be expressed as

$$
F^{e}(x, y)=I^{e}(x, y) * \eta(x, y),
$$

where $I^{e}$ denotes the experimental two-dimensional (2D) intensity at detector pixel $(x, y) . I^{e}$ is the sum of the sample

TABLE II. Theoretical and experimental energetics of benzaldehyde and acetophenone: excited states.

\begin{tabular}{|c|c|c|c|c|c|c|c|}
\hline & & \multicolumn{3}{|c|}{ Benzaldehyde } & \multicolumn{3}{|c|}{ Acetophenone } \\
\hline & & Expt. $^{\mathrm{a}}$ & $\mathrm{MCSCF}^{\mathrm{b}}$ & MCQDPT $^{\mathrm{b}}$ & Expt. $^{c}$ & $\mathrm{MCSCF}^{\mathrm{b}}$ & MCQDPT $^{b}$ \\
\hline$S_{0}$ & & 0 & 0 & 0 & 0 & 0 & 0 \\
\hline$S_{1}$ & $\left({ }^{1} n \pi^{*}\right)$ & 26921 & 26541 & 27370 & 27279 & $\begin{array}{l}\ldots \\
(26703)^{d}\end{array}$ & $\begin{array}{l}\ldots \\
(27278)^{d}\end{array}$ \\
\hline$S_{2}$ & $\left({ }^{1} \pi \pi^{*}\right)$ & 35191 & 35978 & 33028 & 35402 & 36074 & 33329 \\
\hline$T_{1}$ & $\left({ }^{3} n \pi^{*}\right)$ & 25183 & $\begin{array}{l}24844 \\
(24766)^{d}\end{array}$ & $\begin{array}{l}24976 \\
(24899)^{d}\end{array}$ & 25791 & $\begin{array}{l}25556 \\
(25542)^{d}\end{array}$ & $\begin{array}{l}25622 \\
(25514)^{d}\end{array}$ \\
\hline$T_{2}$ & $\left({ }^{3} \pi \pi^{*}\right)$ & & 24821 & 25148 & & 25102 & 25643 \\
\hline$T_{3}$ & $\left({ }^{3} \pi \pi^{*}\right)$ & & & & & 36361 & 32639 \\
\hline
\end{tabular}

${ }^{\mathrm{a}}$ For benzaldehyde, the $S_{1}$ origins are $26919 \mathrm{~cm}^{-1}$ (Ref. 8) $26920 \mathrm{~cm}^{-1}$ (Ref. 19), and $26921 \mathrm{~cm}^{-1}$ (Ref. 17). The $S_{2}$ origins are $35200 \mathrm{~cm}^{-1}$ (Ref. 11) and $35191 \mathrm{~cm}^{-1}$ (Ref. 13). The $S_{3}$ origin is $41334 \mathrm{~cm}^{-1}$ (Ref. 26). The $T_{1}$ origins are $25180 \mathrm{~cm}^{-1}$ (Ref. 18), $397 \mathrm{~nm}$ (Ref. 10), $25184 \mathrm{~cm}^{-1}$ (Ref. 9), $25183 \mathrm{~cm}^{-1}$ (Ref. 8), $397 \mathrm{~nm}$ (Ref. 19), and $25183 \mathrm{~cm}^{-1}$ (Ref. 17).

${ }^{\mathrm{b}} \mathrm{MCSCF}$ and MCQDPT calculations were performed using the 6-311G $(d, p)$ basis set and an active space of ten electrons partitioned in nine orbitals.

${ }^{c}$ For acetophenone, the $S_{1}$ origin is $27279 \mathrm{~cm}^{-1}$ (Ref. 8). The $S_{2}$ origins are $35402 \mathrm{~cm}^{-1}$ (Ref. 43), and $34010 \mathrm{~cm}^{-1}$ (Ref. 6). The $S_{3}$ origin is $41695 \mathrm{~cm}^{-1}$ (Ref. 26). The $T_{1}$ origins are $25791 \mathrm{~cm}^{-1}$ (Ref. 8), and $25786 \mathrm{~cm}^{-1}$ (Ref. 44)

${ }^{\mathrm{d}}$ Values in parenthesis are for structures which the planar symmetry is imposed and results in an imaginary frequency. 
scattering signal $(I)$ and the background scattering $\left(I^{b}\right)$. The latter, in principle, is the result of scattering due to the background gas $\left(I^{a}\right)$, laser $\left(I^{l}\right)$, and the detector $\left(I^{d}\right)$. In order to eliminate the filter function, we simply measure the scattering of monoatomic $\mathrm{Xe}, F_{\mathrm{Xe}}^{e}(x, y)$, which generates a smooth monotonic signal, and measure the contribution of the detector's noise, $F^{d}(x, y)$, which is recorded in the absence of sample and the laser. It is then straightforward to eliminate the filter function by defining the following ratio:

$$
R^{e}(x, y)=\frac{F^{e}(x, y)-F^{d}(x, y)}{F_{\mathrm{Xe}}^{e}(x, y)-F^{d}(x, y)}=\frac{I^{e}(x, y)-I^{d}(x, y)}{I_{\mathrm{Xe}}^{e}(x, y)-I^{d}(x, y)} .
$$

The $2 \mathrm{D}$ ratio patterns, $R^{e}(x, y)$, are converted to onedimensional (1D) intensity ratio curves, $R^{e}(s)$, by radial averaging after locating the center position of the diffraction rings; $s$ denotes the momentum transfer parameter which is related to the distance from the center position, as discussed below [see Eq. (4)]. It follows that

$$
I^{e}(s) \cong R^{e}(s) \times I_{\mathrm{Xe}}^{t}(s)=I(s)+I_{B}^{e}(s),
$$

where $I_{B}^{e}(s)$ is the sum of $I^{a}(s)$ and $I^{l}(s)$. We note that $I_{\mathrm{Xe}}^{t}(s)$ is the simulated 1D atomic scattering of Xe, and Eq. (3) is valid because the atomic scattering of xenon is much larger than its background gas scattering. Below we shall see how $I^{e}(s)$ produces the molecular scattering function, which contains all the structural information.

\section{B. Basic theory}

The theory of gas-phase electron diffraction is well established and described in the literature (see, for example, Ref. 66). Here, for reference, it will only be briefly summarized. Typically, scattering intensity is expressed in terms of the momentum transfer parameter, $s$, between the incident and scattered electrons. For elastic scattering, the $s$ value at each pixel point becomes

$$
s=\left|k_{0}-k\right|=2\left|k_{0}\right| \sin (\theta / 2)=\frac{4 \pi}{\lambda} \sin (\theta / 2),
$$

where $\theta$ is the scattering angle, $\theta=\arctan (d / L), d$ is the distance from the center position, $L$ is camera distance, and $\lambda$ is the de Broglie wavelength of the electron. For a given kinetic energy, $T, \lambda$ becomes

$$
\lambda=\frac{h c}{p c}=\frac{h c}{\sqrt{T\left(T+2 m_{0} c^{2}\right)}},
$$

where $h$ is Planck's constant, $m_{0}$ is the rest mass of an electron, and $c$ is the speed of light. With $T$ at $30 \mathrm{keV}$ for an electron experiencing a $30 \mathrm{kV}$ acceleration voltage, $\lambda$ is $0.06979 \AA$. The velocity of the electron at a given kinetic energy is given by

$$
v=c \sqrt{1-\left(\frac{m_{0} c^{2}}{T+m_{0} c^{2}}\right)^{2}}
$$

In the independent atomic model, it is assumed that the electronic potential of a molecule is equivalent to an assembly of unperturbed atomic potentials at each appropriate nuclear position within that molecule. Hence, electron scat- tering from a molecule is simply the sum of each individual atomic contribution and each interatomic molecular interference contribution. The total scattering intensity, $I(s)$, can be written as

$$
I(s)=I_{A}(s)+I_{M}(s) .
$$

The atomic scattering, $I_{A}(s)$, is a sum of elastic and inelastic components for each atom;

$$
I_{A}(s)=\sum_{i} f_{i}^{2}+4 \frac{S_{i}}{a_{0}^{2} s^{4}}
$$

where $f_{i}$ and $S_{i}$ are the elastic and the inelastic scattering amplitudes, respectively, for the ith nucleus and $a_{0}$ is the bohr radius. Theoretical values of scattering and phase factors are available from the literature. ${ }^{67}$ The molecular term, $I_{M}(s)$, contains the desired structural information and is written for an isotropic distribution of molecules as

$$
I_{M}(s)=\sum_{i, j} f_{i} f_{j} \cos \left(\eta_{i}-\eta_{j}\right)\left\langle\frac{\sin \left(s r_{i j}\right)}{s r_{i j}}\right\rangle_{\text {vib }},
$$

where $\eta_{i}$ is the phase factor for the ith nucleus, $r_{i j}$ is the distance between $i$ th and $j$ th nuclei, and the bracket denotes an average over the vibrational motion. Using the harmonic oscillator approximation, Eq. (9) becomes

$$
I_{M}(s)=\sum_{i, j} f_{i} f_{j} \cos \left(\eta_{i}-\eta_{j}\right) \exp \left(-\frac{1}{2} l_{h}^{2} s^{2}\right) \frac{\sin \left(s r_{a, i j}\right)}{s r_{e, i j}}
$$

where $r_{e, i j}$ and $r_{a, i j}$ are the equilibrium and the effective internuclear distances between $i$ th and $j$ th nuclei, respectively, and $l_{h}$ is the harmonic mean vibrational amplitude. By accounting for the small perturbation by anharmonicity, the effective distance can be expressed by

$$
\begin{aligned}
& r_{a}=r_{g}-\frac{l_{h}^{2}}{r_{e}}, \\
& r_{g}=r_{e}+\frac{3}{2} a l_{h}^{2},
\end{aligned}
$$

where $a$ is the anharmonicity constant. $r_{g}$ corresponds to the distance between centers of gravity at temperature $T$. Often $a$ is set at 2 for direct bonds and at 0 for nonbonded distances. Empirical formulas are used to estimate the harmonic mean vibrational amplitudes, $l_{h}$, at room temperature ${ }^{68}$ which are then scaled to the experimental temperature. ${ }^{66}$

To visualize the molecular structural parameters as well as emphasize the damped oscillatory behavior, the modified molecular scattering function, $s M(s)$, is defined as

$$
s M(s)=s \frac{I_{M}(s)}{f_{I} f_{J}},
$$

where $f_{I}$ and $f_{J}$ are atomic scattering factors of the selected nuclei. Sine Fourier transformation then provides the radial distribution, $f(r)$, 


$$
\begin{aligned}
f(r) & =\int_{0}^{s_{\max }} s M(s) \sin (s r) \exp \left(-k s^{2}\right) d s \\
& \approx \sum_{k} s M\left(s_{k}\right) \sin \left(s_{k} r\right) \exp \left(-k s_{k}^{2}\right) \Delta s_{k},
\end{aligned}
$$

which displays the internuclear distance information in an intuitive form. The Gaussian window function $\exp \left(-k s^{2}\right)$ is included to account for the finite range of data acquired. Typically, $k=0.005 \AA^{2}$ for our $s$ range from 2 to $16 \AA^{-1}$. A piece of the theoretical $s M(s)$ is appended to the low $s$ portion of the experimental $s M(s)$ to provide continuity required by the transform. It is to be noted that for a pair of nuclei $i$ and $j$, the area under its corresponding peak in the $f(r)$ is proportional to $n_{i j} Z_{i} Z_{j} / r_{i j}$ where $n_{i j}$ is the multiplicity of the distance $r_{i j}$ in the molecule. This can be seen from

$$
f(r) \propto \sum_{i, j} \frac{Z_{i} Z_{j}}{r_{e, i j} \sqrt{2 k+l_{i j}^{2}}} \exp \left[-\frac{\left(r-r_{a, i j}\right)^{2}}{2\left(2 k+l_{i j}^{2}\right)}\right],
$$

where the Gaussian function contains the convolution of both the $k$ damping and $l$ vibrational amplitude (see Refs. 69 and 70). Although the above treatment is for an isotropic distribution, it has been shown elsewhere ${ }^{71}$ that orientational effects in UED can be quantitatively expressed.

\section{Refining static diffraction data}

The experimentally obtained scattering intensity, $I^{e}(s)$, contains the molecular and atomic scatterings as well as any background, $I_{B}^{e}$, that results from background gas (smoothly varying) and laser scattering (minor), as discussed above. Accordingly,

$$
I^{e}(s) \cong I_{M}^{e}(s)+I_{A}^{e}(s)+I_{B}^{e}(s) .
$$

From the atomic scattering factors, known in the literature, ${ }^{67}$ and the polynomial fit to $I_{B}^{e}(s)$, we can obtain the molecular scattering intensity. The experimental modified molecular scattering function becomes

$$
s M^{e}(s)=s \frac{I_{M}^{e}(s)}{f_{I} f_{J}}
$$

while the theoretical modified molecular scattering function is defined as

$$
s M^{t}(s)=s \frac{I_{M}^{t}(s)}{f_{I} f_{J}} .
$$

Structural refinement is carried out in the modified molecular scattering space by minimizing the difference between the experimental and theoretical $s M(s)$ curves. The difference, $\chi^{2}$, is defined as

$$
\chi^{2}=\sum_{s}\left\{\frac{\left(s M^{e}-s M^{t}\right)}{\sigma_{s M^{e}}}\right\}^{2},
$$

where $\sigma_{s M^{e}}=s\left(\sigma_{I^{e}} / f_{I} f_{J}\right.$. It is to be noted that Eq. (19) reduces to

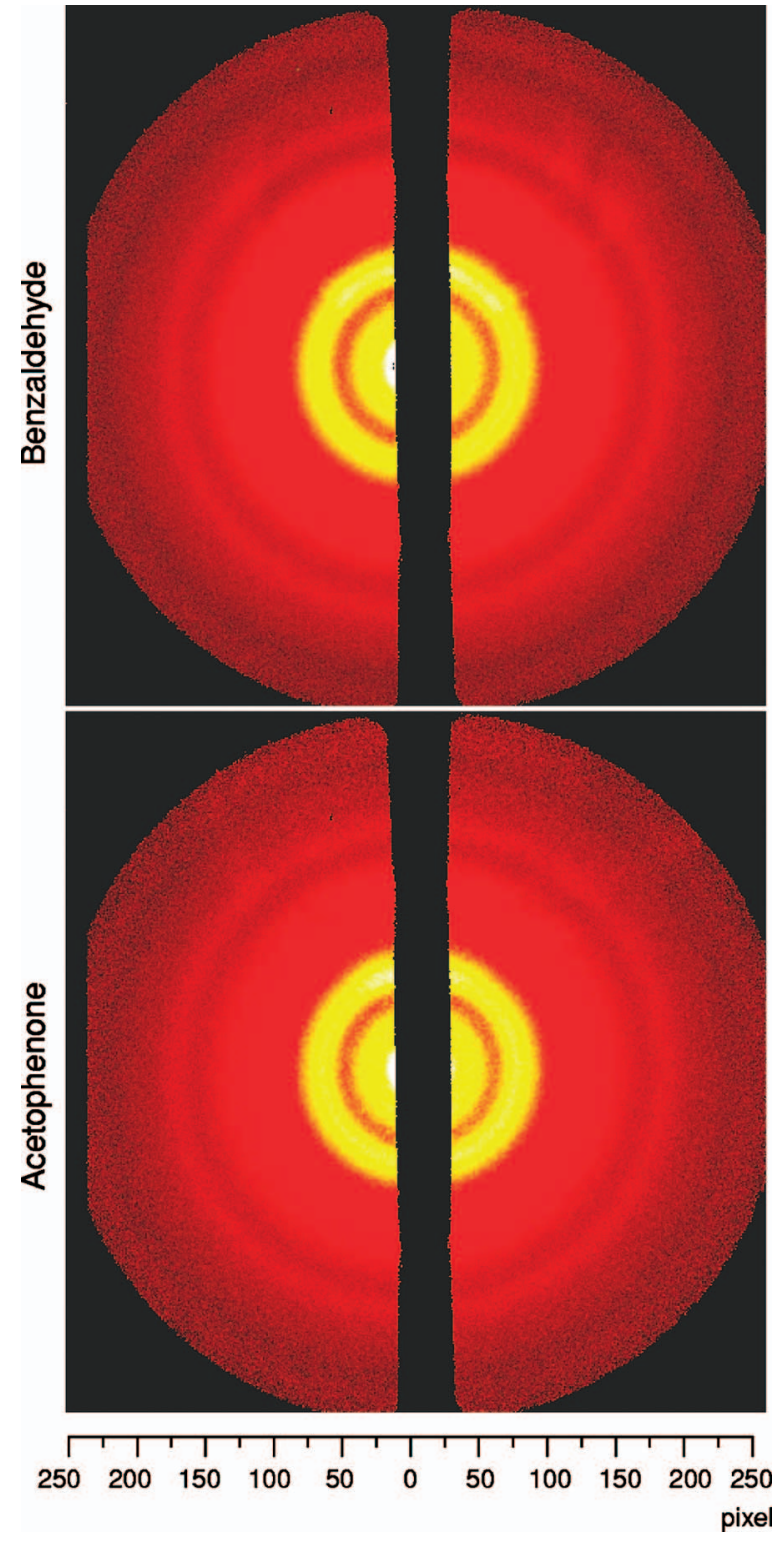

FIG. 5. (Color) Two-dimensional diffraction ratio patterns for benzaldehyde and acetophenone in their ground states.

$$
\chi^{2}=\sum_{s}\left\{\frac{I_{M}^{e}-I_{M}^{t}}{\sigma_{I^{e}}}\right\}^{2}
$$

and therefore refinement is unaffected by conversion to $s M(s)$ from the raw intensity space.

The normal equation method (see Ref. 72 and references therein) is employed for linear parameters (scaling factor, polynomial background, and mixture fractions, if any) and the Levenberg-Marquardt algorithm is invoked for nonlinear (structural and vibrational) parameters. $\chi^{2}$ was primarily used to quantify the fit, although $R$, a standard goodness-offit parameter in electron diffraction, was also checked throughout the refinement.

$$
R=\sqrt{\frac{\sum_{s}\left\{\left(s M^{e}-s M^{t}\right) / \sigma_{\left.s M^{e}\right\}^{2}}\right.}{\sum_{s}\left(s M^{t} / \sigma_{s M^{e}}\right)^{2}} .}
$$

As noted, the difference between $\chi^{2}$ [Eq. (19)] and $R$ [Eq. (21)] is in the division by the theoretical term of 
TABLE III. The refined structures of ground state benzaldehyde and acetophenone.

\begin{tabular}{|c|c|c|c|c|}
\hline Molecule & Species & Parameters & Refined value $^{\mathrm{a}}$ & Theor. $^{\text {b }}$ \\
\hline \multirow[t]{16}{*}{ Benzaldehyde $^{c}$} & \multirow[t]{16}{*}{$S_{0}$} & $\mathrm{C} 1-\mathrm{C} 2, \mathrm{C} 6-\mathrm{C} 1$ & $1.388 \pm 0.004$ & $1.401,1.398$ \\
\hline & & $\mathrm{C} 2-\mathrm{C} 3, \mathrm{C} 5-\mathrm{C} 6$ & $1.381 \pm 0.004$ & $1.388,1.392$ \\
\hline & & $\mathrm{C} 3-\mathrm{C} 4, \mathrm{C} 4-\mathrm{C} 5$ & $1.417^{\mathrm{d}}$ & $1.398,1.394$ \\
\hline & & $\mathrm{C} 1-\mathrm{C} 7$ & $1.480 \pm 0.005$ & 1.481 \\
\hline & & $\mathrm{C} 7-\mathrm{O} 8$ & $1.200 \pm 0.002$ & 1.209 \\
\hline & & $\mathrm{C} 6-\mathrm{C} 1-\mathrm{C} 2$ & $120.0 \pm 0.7$ & 119.9 \\
\hline & & $\mathrm{C} 1-\mathrm{C} 2-\mathrm{C} 3, \mathrm{C} 5-\mathrm{C} 6-\mathrm{C} 1$ & $121.0 \pm 0.9$ & $119.9,120.2$ \\
\hline & & $\mathrm{C} 2-\mathrm{C} 3-\mathrm{C} 4, \mathrm{C} 4-\mathrm{C} 5-\mathrm{C} 6$ & $119.1^{\mathrm{d}}$ & $120.0,119.7$ \\
\hline & & $\mathrm{C} 3-\mathrm{C} 4-\mathrm{C} 5$ & $119.9^{\mathrm{d}}$ & 120.3 \\
\hline & & $\mathrm{C} 2-\mathrm{C} 1-\mathrm{C} 7$ & $120.0^{\mathrm{d}}$ & 120.3 \\
\hline & & $\mathrm{C} 1-\mathrm{C} 7-\mathrm{O} 8$ & $126.4 \pm 0.3$ & 124.9 \\
\hline & & $\mathrm{H}-\mathrm{C} 7$ & & 1.112 \\
\hline & & $\mathrm{H}-\mathrm{C} 7-\mathrm{O} 8$ & & 120.7 \\
\hline & & $\mathrm{H}-\mathrm{C}_{\text {ring }}$ & & $1.084-1.086$ \\
\hline & & $\mathrm{H}-\mathrm{C}_{\text {ring }}-\mathrm{C}_{\text {ring }}$ & & $118.5-120.2$ \\
\hline & & $\mathrm{C} 2-\mathrm{C} 1-\mathrm{C} 7-\mathrm{O} 8$ & & 0.0 \\
\hline \multirow[t]{19}{*}{ Acetophenone $^{c}$} & \multirow[t]{19}{*}{$S_{0}$} & $\mathrm{C} 1-\mathrm{C} 2, \mathrm{C} 6-\mathrm{C} 1$ & $1.407 \pm 0.017$ & $1.401,1.400$ \\
\hline & & $\mathrm{C} 2-\mathrm{C} 3, \mathrm{C} 5-\mathrm{C} 6$ & $1.401 \pm 0.019$ & $1.388,1.393$ \\
\hline & & $\mathrm{C} 3-\mathrm{C} 4, \mathrm{C} 4-\mathrm{C} 5$ & $1.380^{\mathrm{d}}$ & $1.396,1.393$ \\
\hline & & $\mathrm{C} 1-\mathrm{C} 7$ & $1.488 \pm 0.034$ & 1.502 \\
\hline & & $\mathrm{C} 7-\mathrm{O} 8$ & $1.198 \pm 0.003$ & 1.215 \\
\hline & & $\mathrm{C} 7-\mathrm{C} 9$ & $1.548 \pm 0.016$ & 1.519 \\
\hline & & $\mathrm{C} 6-\mathrm{C} 1-\mathrm{C} 2$ & $118.7 \pm 1.4$ & 119.1 \\
\hline & & $\mathrm{C} 1-\mathrm{C} 2-\mathrm{C} 3, \mathrm{C} 5-\mathrm{C} 6-\mathrm{C} 1$ & $120.0 \pm 1.1$ & $120.5,120.4$ \\
\hline & & $\mathrm{C} 2-\mathrm{C} 3-\mathrm{C} 4, \mathrm{C} 4-\mathrm{C} 5-\mathrm{C} 6$ & $120.7^{\mathrm{d}}$ & $120.0,120.0$ \\
\hline & & $\mathrm{C} 3-\mathrm{C} 4-\mathrm{C} 5$ & $120.0^{\mathrm{d}}$ & 120.0 \\
\hline & & $\mathrm{C} 2-\mathrm{C} 1-\mathrm{C} 7$ & $120.5^{\mathrm{d}}$ & 118.1 \\
\hline & & $\mathrm{C} 1-\mathrm{C} 7-\mathrm{O} 8$ & $124.1 \pm 0.4$ & 120.6 \\
\hline & & $\mathrm{C} 1-\mathrm{C} 7-\mathrm{C} 9$ & $116.0 \pm 0.4$ & 118.8 \\
\hline & & $\mathrm{H}-\mathrm{C}_{\text {ring }}$ & & $1.083-1.084$ \\
\hline & & $\mathrm{H}-\mathrm{C}_{\text {ring }}-\mathrm{C}_{\text {ring }}$ & & $120.0-120.1$ \\
\hline & & $\mathrm{H}-\mathrm{C} 9$ & & $1.089-1.094$ \\
\hline & & $\mathrm{H}-\mathrm{C} 9-\mathrm{C} 7$ & & $108.5-111.0$ \\
\hline & & $\mathrm{H}-\mathrm{C} 9-\mathrm{C} 7-\mathrm{O} 8$ & $29.5 \pm 3.0$ & 0.0 \\
\hline & & $\mathrm{C} 2-\mathrm{C} 1-\mathrm{C} 7-\mathrm{O} 8$ & & 0.0 \\
\hline
\end{tabular}

The error bars reported here are $3 \sigma$.

${ }^{\mathrm{b}}$ Theoretical structures were obtained at B3LYP/6-311G $(d, p)$ for the ground state of benzaldehyde and acetophenone.

${ }^{\mathrm{c}} C_{2 v}$ symmetry was imposed for the phenyl ring.

${ }^{\mathrm{d}}$ Dependent variables.

$\Sigma_{s}\left(s M^{t} / \sigma_{s M^{e}}\right)^{2}$, making $R$ a relative quality of fit parameter. Typically in the analyses we minimize $\chi^{2}$ and the smaller the $R$ value, the better the agreement; for ground state structural analyses $R$ is typically $<0.05$, very similar to conventional gas-phase electron diffraction (GED), while for transient structures $R$ is significantly larger.

\section{UED diffraction frame reference}

Upon irradiation of the molecular beam by the excitation laser, a fraction of the molecules will absorb and undergo photophysical and/or photochemical processes, while most do not interact with the photons and will remain in their initial state throughout the experiment. Molecules absorbing a photon will undergo an electronic transition and possibly structural changes. With this in mind, the scattering intensity at a given time point, $t$, can be expressed as a sum of scattering intensities for all possible configurations, $\alpha$.

$$
I(s ; t)=\sum_{\alpha} I_{\alpha}(s ; t)=\sum_{\alpha} f_{\alpha}(t) I_{\alpha}(s),
$$

where $f_{\alpha}(t)$ is the fractional contribution of $I_{\alpha}(s)$ at time $t$. This total intensity has a significant component of unreacted, unperturbed molecules in their initial states.

The reacting molecules can be highlighted using the frame-referencing method. Diffraction data from before excitation (reference data) are subtracted from diffraction data at time $t$ after excitation. The frame-difference data eliminate essentially all unreacted species as well as the atomic scattering and systematic background, thereby emphasizing the structural changes (and temporal evolution of those structures) during the reaction. For example, for $t_{\text {ref }}<0$, 

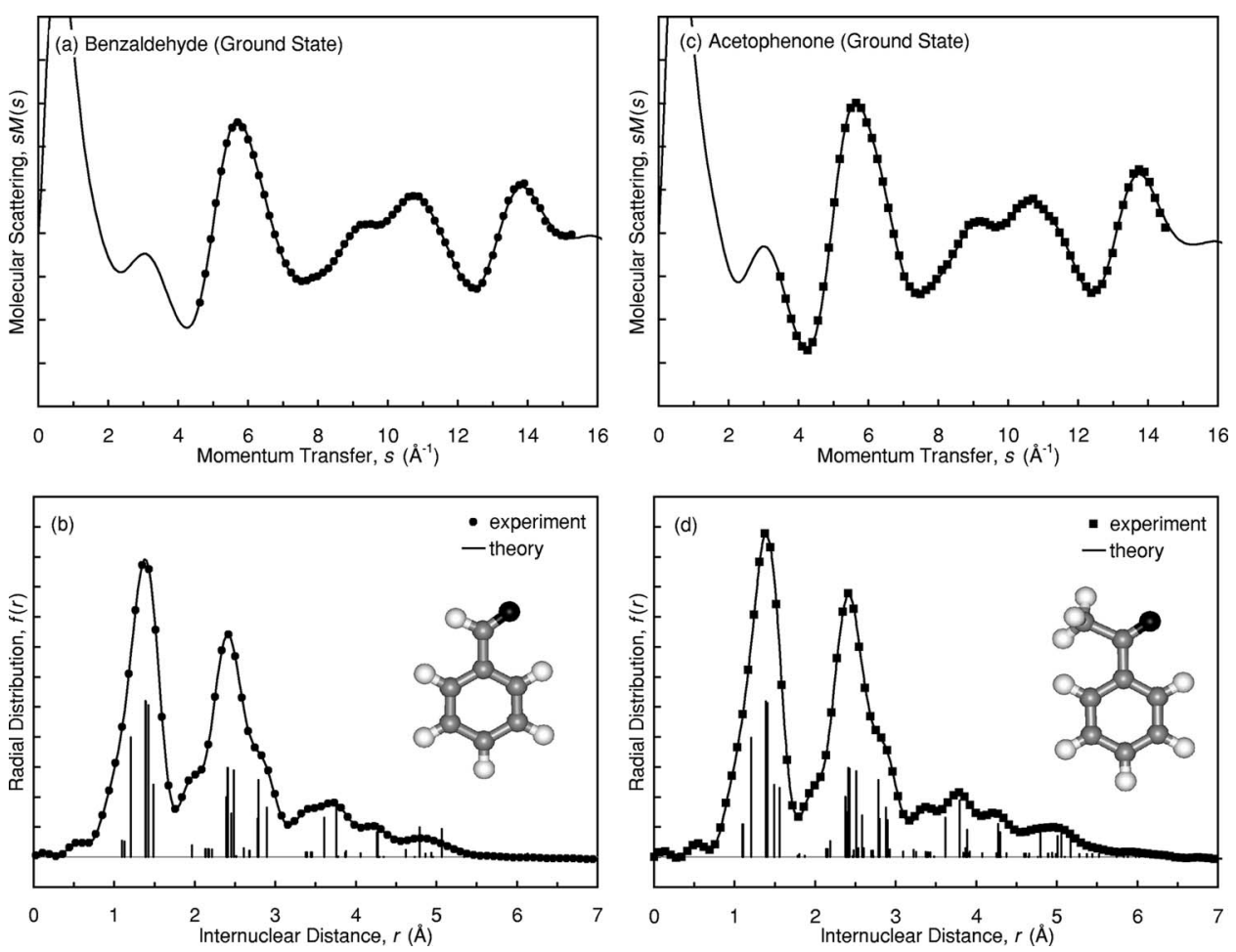

FIG. 6. Modified molecular scattering curves, $s M(s)$, and radial distributions, $f(r)$, for ground state benzaldehyde and acetophenone. The experimental (filled symbols) and theoretical (solid line) of benzaldehyde [(a) and (b) $R=0.031$ and acetophenone [(c) and (d) $R=0.028$ in their ground states, together with the refined molecular structures.

$$
\begin{aligned}
\Delta I(s ; t)= & I(s ; t)-I\left(s ; t_{\mathrm{ref}}\right) \\
= & \sum_{\alpha} f_{\alpha}(t) I_{\alpha}(s)-1 \times I_{r}(s) \\
= & {\left[f_{r}(t) I_{r}(s)+\sum_{\alpha \neq r}\left\{f_{\alpha}(t) I_{\alpha}(s)\right\}\right] } \\
& -\left\{f_{r}(t)+\sum_{\alpha \neq r} f_{\alpha}(t)\right\} I_{r}(s) \\
= & \sum_{\alpha \neq r}\left\{f_{\alpha}(t) I_{\alpha}(s)\right\}-\left\{\sum_{\alpha \neq r} f_{\alpha}(t)\right\} I_{r}(s) \\
= & \sum_{\alpha \neq r} f_{\alpha}(t)\left\{I_{\alpha}(s)-I_{r}(s)\right\},
\end{aligned}
$$

where the $r$ subscript refers to the reference. In this case, because the structure of the reference (ground state) has already been determined, Eq. (23) provides the structures of the intermediates/products and their fractions. $\Delta s M^{e}(s ; t)$ can then be calculated in the same fashion as $s M^{e}(s)$ was above. The fractional and structural refinements of products and intermediates are carried out with the same nonlinear fitting; minimizing $\chi^{2}$ in Eq. (19) with $\Delta s M^{e}(s ; t)$ being substituted for $s M^{e}(s)$ at each time point. A positive time point could also be used as the reference frame in order to enhance the signal of a particular transient structure, as shown elsewhere. ${ }^{55}$

\section{E. Structural refinement and assessment of errors}

For each datum, we typically collect 100 diffraction patterns that each consist of 240000 electron pulse shots (see Sec. III E). These are then averaged to obtain the averaged raw diffraction patterns, $\bar{F}^{e}$ (sample), $\bar{F}^{d}$ (detector background), and $\bar{F}_{\mathrm{Xe}}^{e}$ (xenon). The experimental ratio pattern, $\bar{R}^{e}$, is then generated with Eq. (2) using these averaged patterns.
The error for each pixel of the averaged patterns is then discarded. The 2D ratio patterns, $\bar{R}^{e}(x, y)$, are converted into 1D intensity ratio curves, $\bar{R}^{e}(s)$, by radial average. The error in the radial averaging is evaluated by "the standard deviation of the mean" as follows: ${ }^{73}$

$$
\sigma_{R^{e}}^{-}(s)=\frac{\sqrt{\sum_{r(x, y)=d}\left\{\bar{R}^{e}(x, y)-\bar{R}^{e}(s)\right\}^{2}}}{n_{r}},
$$

where $n_{r}$ is the number of pixels in the 2D pattern at a given distance, $d$, from the center. The experimental error is evaluated by $\sigma_{I^{e}}(s)=\sigma_{R^{e}}{ }^{2}(s) \times I_{\mathrm{Xe}}^{t}(s)$. During the refinement, the derivative of $\chi^{2}$ with respect to each parameter $\varphi$ is utilized to find a minimum position.

$$
\beta \equiv-\frac{1}{2} \frac{\partial \chi^{2}}{\partial \varphi}=0
$$

The estimated covariance matrix of the standard errors in the fitted parameters becomes

$$
[C] \equiv[\alpha]^{-1}=\left[\frac{1}{2} \frac{\partial^{2}\left(\chi^{2}\right)}{\partial \varphi_{i} \partial \varphi_{j}}\right]^{-1},
$$

from which the variance associated with the estimated parameter $\varphi_{i}$ is $\sigma^{2}\left(\varphi_{i}\right)$ and equals $C_{i i}$. The error associated with the estimated parameter is

$$
\delta\left(\varphi_{i}\right)=\sqrt{\Delta \chi_{\nu}^{2}} \sqrt{\sigma^{2}\left(\varphi_{i}\right)}
$$

where $\Delta \chi_{\nu}^{2}$ is a coefficient determined from the confidence level and the degrees of freedom of the fit, $\nu$; for a table of $\Delta \chi_{\nu}^{2}$ see Ref. 72 . 

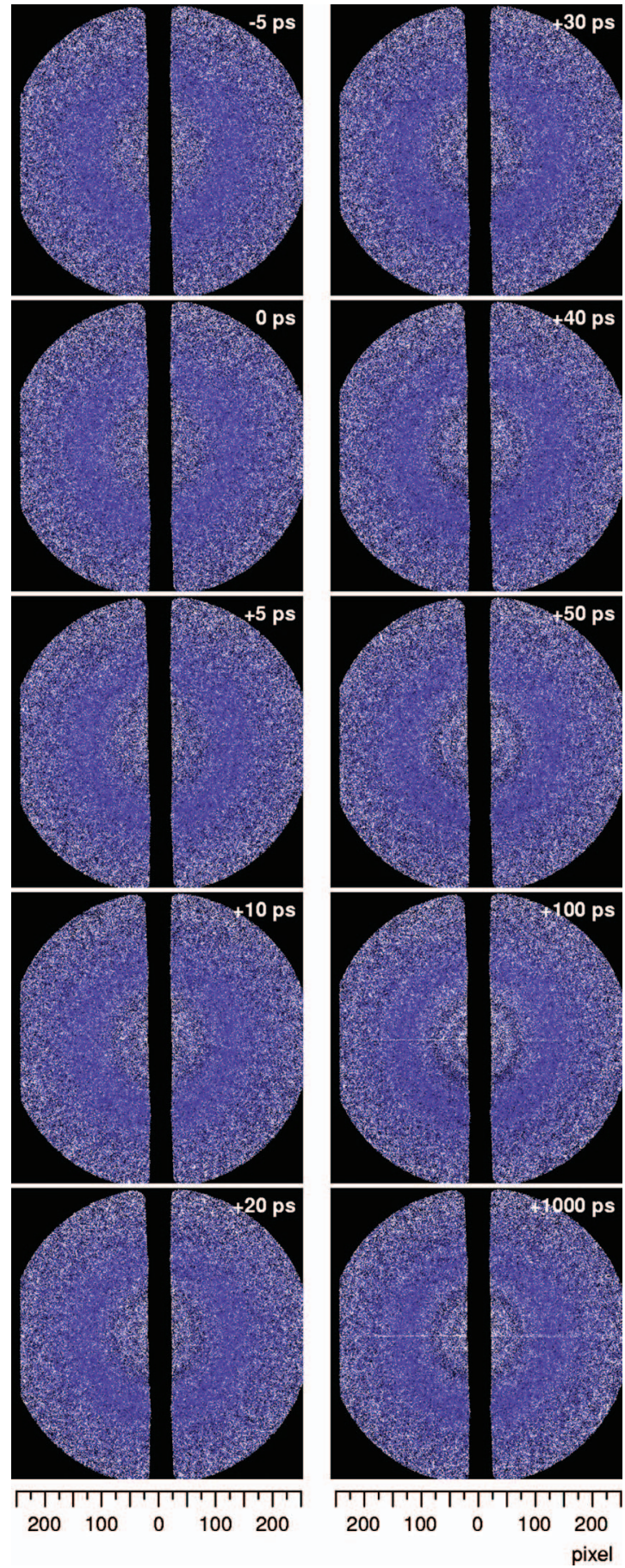

FIG. 7. (Color) Frame-referenced patterns for time-resolved benzaldehyde structural dynamics. The reference frame is at $t=-100 \mathrm{ps}$, before the arrival of the excitation laser pulse.

\section{RESULTS}

For all results presented, the camera distance was measured independently by recording electron diffraction data from carbon dioxide. Since the molecular structure of carbon dioxide is well known from the literature ${ }^{74}$ the camera distance could be accurately established. For the studies presented here, the camera distances were determined to be $13.38 \mathrm{~cm}$ (benzaldehyde studies) and $13.42 \mathrm{~cm}$ (acetophenone studies).
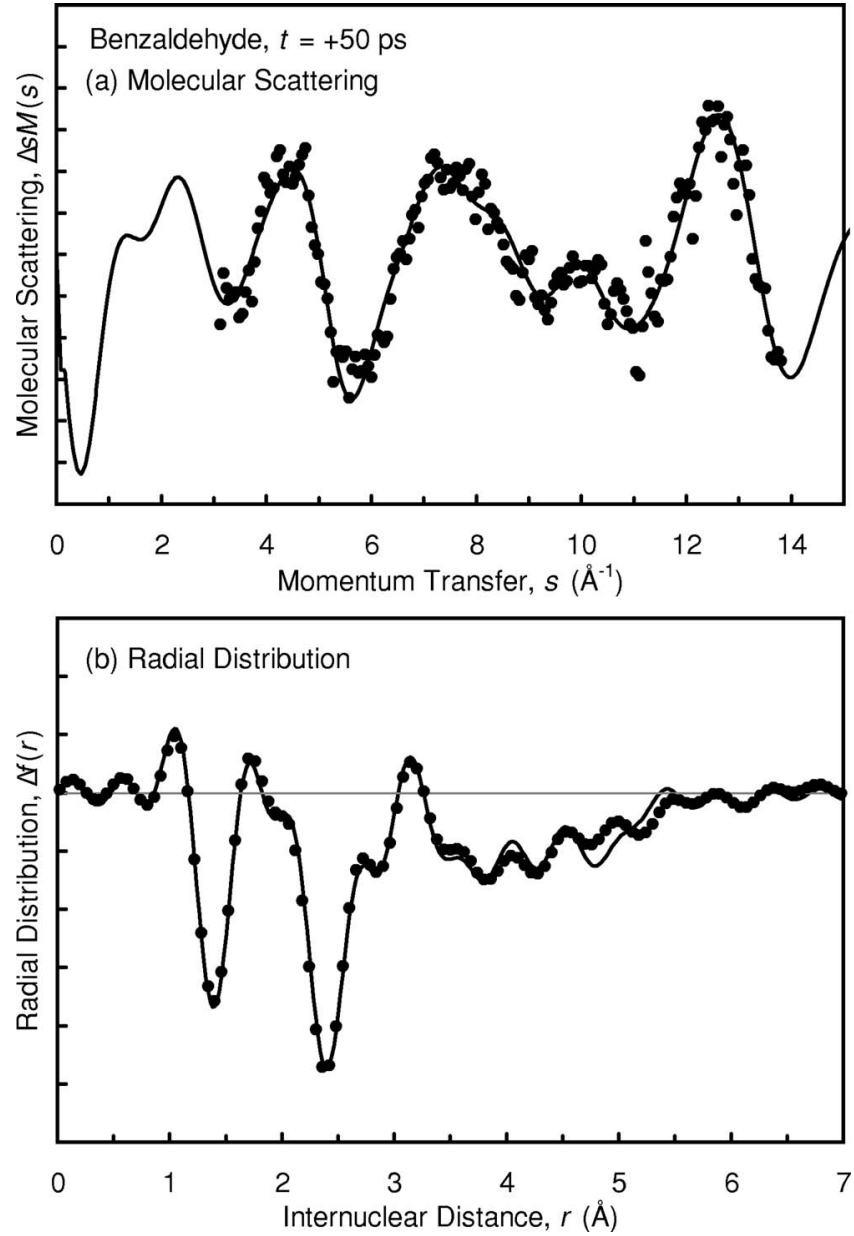

FIG. 8. Modified molecular scattering curves, $\Delta s M(s ; t)$, and difference radial distributions, $\Delta f(r ; t)$ for benzaldehyde at $t=+50 \mathrm{ps}$ upon $266.7 \mathrm{~nm}$ excitation. The experimental data points are shown as filled symbols and the refined theory is a solid line. $(R=0.399)$.

\section{A. Ground state structures: Benzaldehyde and acetophenone}

For benzaldehyde, the two-dimensional ratio pattern of the ground state diffraction is shown in Fig. 5. Raw data were converted to $s M(s)$ format and compared with the initial guess structure derived from the DFT calculations. The resulting $R$ value is 0.043 . The mean amplitudes of vibration were obtained from empirical formulas ${ }^{68}$ and extrapolated to $483 \mathrm{~K}$ using the nozzle temperature as the temperature of the sample. The polynomial background and theory scaling factor were optimized for the best fit. The data range used for refinement was $s=4.7-15.4 \AA^{-1}$, the center of the detector's range.

The structure of benzaldehyde was subsequently refined. As described by Borisenko et al. in a previous electron diffraction experiment on benzaldehyde, ${ }^{33}$ a $C_{2 v}$ model was used for the aryl ring to simplify the refinement; our DFT calculation predicts that the ring is nearly of $C_{2 v}$ symmetry. The refined ground state benzaldehyde structural parameters $(R=0.031)$ are listed in Table III. Refined bond distances and angles are within $0.01 \AA$ and $1^{\circ}$, respectively, of the structure derived by Borisenko et al. The carbonyl torsional angle was tested in preliminary fitting and found to remain nearly planar. For the final refinement, it was fixed in the plane of the 
TABLE IV. The refined structures of physical and chemical products.

\begin{tabular}{|c|c|c|c|c|}
\hline Molecule & Species & Parameters & Refined value $^{\mathrm{a}}$ & Theor. $^{\text {b }}$ \\
\hline \multirow[t]{21}{*}{ Benzaldehyde } & Carbon monoxide & $\mathrm{C}-\mathrm{O}$ & & 1.127 \\
\hline & \multirow[t]{4}{*}{ Benzene } & $\mathrm{C}-\mathrm{C}$ & \multirow[t]{4}{*}{$1.371 \pm 0.004$} & 1.393 \\
\hline & & $\mathrm{C}-\mathrm{H}$ & & 1.084 \\
\hline & & $\mathrm{C}-\mathrm{C}-\mathrm{C}$ & & 120.0 \\
\hline & & $\mathrm{H}-\mathrm{C}-\mathrm{C}$ & & 120.0 \\
\hline & \multirow[t]{16}{*}{ Quinoid triplet $^{\mathrm{c}}$} & $\mathrm{C} 1-\mathrm{C} 2, \mathrm{C} 6-\mathrm{C} 1$ & $1.479 \pm 0.029$ & $1.482,1.474$ \\
\hline & & $\mathrm{C} 2-\mathrm{C} 3, \mathrm{C} 5-\mathrm{C} 6$ & $1.322 \pm 0.029$ & $1.362,1.358$ \\
\hline & & $\mathrm{C} 3-\mathrm{C} 4, \mathrm{C} 4-\mathrm{C} 5$ & $1.487^{\mathrm{d}}$ & $1.442,1.460$ \\
\hline & & $\mathrm{C} 1-\mathrm{C} 7$ & $1.420 \pm 0.045$ & 1.421 \\
\hline & & C7-O8 & $1.263 \pm 0.031$ & 1.237 \\
\hline & & $\mathrm{C} 6-\mathrm{C} 1-\mathrm{C} 2$ & $115.6 \pm 6.2$ & 117.8 \\
\hline & & $\mathrm{C} 1-\mathrm{C} 2-\mathrm{C} 3, \mathrm{C} 5-\mathrm{C} 6-\mathrm{C} 1$ & $122.3 \pm 7.7$ & $120.3,120.7$ \\
\hline & & $\mathrm{C} 2-\mathrm{C} 3-\mathrm{C} 4, \mathrm{C} 4-\mathrm{C} 5-\mathrm{C} 6$ & $122.4^{\mathrm{d}}$ & $121.2,120.8$ \\
\hline & & $\mathrm{C} 3-\mathrm{C} 4-\mathrm{C} 5$ & $115.0^{\mathrm{d}}$ & 119.2 \\
\hline & & $\mathrm{C} 2-\mathrm{C} 1-\mathrm{C} 7$ & $122.2^{\mathrm{d}}$ & 120.9 \\
\hline & & $\mathrm{C} 1-\mathrm{C} 7-\mathrm{O} 8$ & $125.4 \pm 2.6$ & 123.1 \\
\hline & & $\mathrm{C} 7-\mathrm{H}$ & & 1.087 \\
\hline & & $\mathrm{H}-\mathrm{C} 7-\mathrm{O} 8$ & & 119.2 \\
\hline & & $\mathrm{H}-\mathrm{C}_{\text {ring }}$ & & $1.072-1.075$ \\
\hline & & $\mathrm{H}-\mathrm{C}_{\text {ring }}-\mathrm{C}_{\text {ring }}$ & & $117.9-120.3$ \\
\hline & & $\mathrm{C} 2-\mathrm{C} 1-\mathrm{C} 7-\mathrm{O} 8$ & & 0.0 \\
\hline \multirow[t]{30}{*}{ Acetophenone } & \multirow[t]{2}{*}{ Methyl } & $\mathrm{C}-\mathrm{H}$ & & 1.080 \\
\hline & & $\mathrm{H}-\mathrm{C}-\mathrm{H}$ & & 120.0 \\
\hline & \multirow[t]{12}{*}{ Benzoyl } & $\mathrm{C} 1-\mathrm{C} 2, \mathrm{C} 6-\mathrm{C} 1$ & $1.403 \pm 0.024$ & $1.403,1.398$ \\
\hline & & $\mathrm{C} 2-\mathrm{C} 3, \mathrm{C} 5-\mathrm{C} 6$ & $1.392^{\mathrm{d}}$ & $1.388,1.391$ \\
\hline & & $\mathrm{C} 3-\mathrm{C} 4, \mathrm{C} 4-\mathrm{C} 5$ & $1.370^{\mathrm{d}}$ & $1.397,1.394$ \\
\hline & & $\mathrm{C} 1-\mathrm{C} 7$ & $1.487 \pm 0.032$ & 1.482 \\
\hline & & C7-O8 & $1.164 \pm 0.009$ & 1.186 \\
\hline & & $\mathrm{C} 6-\mathrm{C} 1-\mathrm{C} 2$ & $120.6 \pm 1.1$ & 120.2 \\
\hline & & $\mathrm{C} 1-\mathrm{C} 2-\mathrm{C} 3, \mathrm{C} 5-\mathrm{C} 6-\mathrm{C} 1$ & $120.1^{\mathrm{d}}$ & $119.7,119.9$ \\
\hline & & $\mathrm{C} 2-\mathrm{C} 3-\mathrm{C} 4, \mathrm{C} 4-\mathrm{C} 5-\mathrm{C} 6$ & $116.2^{\mathrm{d}}$ & $119.9,119.8$ \\
\hline & & $\mathrm{C} 3-\mathrm{C} 4-\mathrm{C} 5$ & $127.0^{\mathrm{d}}$ & 120.5 \\
\hline & & $\mathrm{C} 2-\mathrm{C} 1-\mathrm{C} 7$ & $119.7^{\mathrm{d}}$ & 120.5 \\
\hline & & $\mathrm{C} 1-\mathrm{C} 7-\mathrm{O} 8$ & $130.6 \pm 2.7$ & 128.5 \\
\hline & & $\mathrm{H}-\mathrm{C}_{\text {ring }}$ & 1.084 & 1.084 \\
\hline & \multirow[t]{16}{*}{ Quinoid triplet $^{\mathrm{c}}$} & $\mathrm{C} 1-\mathrm{C} 2, \mathrm{C} 6-\mathrm{C} 1$ & $1.495 \pm 0.014$ & $1.487,1.474$ \\
\hline & & $\mathrm{C} 2-\mathrm{C} 3, \mathrm{C} 5-\mathrm{C} 6$ & $1.349 \pm 0.021$ & $1.358,1.356$ \\
\hline & & $\mathrm{C} 3-\mathrm{C} 4, \mathrm{C} 4-\mathrm{C} 5$ & $1.441^{\mathrm{d}}$ & $1.446,1.462$ \\
\hline & & $\mathrm{C} 1-\mathrm{C} 7$ & $1.460^{\mathrm{d}}$ & 1.446 \\
\hline & & $\mathrm{C} 7-08$ & $1.214 \pm 0.018$ & 1.224 \\
\hline & & $\mathrm{C} 7-\mathrm{C} 9$ & $1.530^{\mathrm{d}}$ & 1.516 \\
\hline & & $\mathrm{C} 6-\mathrm{C} 1-\mathrm{C} 2$ & $117.3 \pm 1.3$ & 117.4 \\
\hline & & $\mathrm{C} 1-\mathrm{C} 2-\mathrm{C} 3, \mathrm{C} 5-\mathrm{C} 6-\mathrm{C} 1$ & $120.7^{\mathrm{d}}$ & $120.7,120.8$ \\
\hline & & $\mathrm{C} 2-\mathrm{C} 3-\mathrm{C} 4, \mathrm{C} 4-\mathrm{C} 5-\mathrm{C} 6$ & $119.7^{\mathrm{d}}$ & $121.1,121.1$ \\
\hline & & $\mathrm{C} 3-\mathrm{C} 4-\mathrm{C} 5$ & $121.9^{\mathrm{d}}$ & 118.8 \\
\hline & & $\mathrm{C} 2-\mathrm{C} 1-\mathrm{C} 7$ & $121.3^{\mathrm{d}}$ & 119.2 \\
\hline & & $\mathrm{C} 1-\mathrm{C} 7-\mathrm{O} 8$ & $126.0 \pm 7.9$ & 119.8 \\
\hline & & $\mathrm{C} 1-\mathrm{C} 7-\mathrm{C} 9$ & $117.0^{\mathrm{d}}$ & 120.4 \\
\hline & & $\mathrm{H}-\mathrm{C}_{\text {ring }}$ & & $1.071-1.075$ \\
\hline & & $\mathrm{H}-\mathrm{C}_{\text {ring }}-\mathrm{C}_{\text {ring }}$ & & $117.6-120.9$ \\
\hline & & $\mathrm{C} 2-\mathrm{C} 1-\mathrm{C} 7-\mathrm{O} 8$ & $0.8 \pm 256$ & 0.0 \\
\hline
\end{tabular}

${ }^{a}$ The error bars reported here are $3 \sigma$.

${ }^{\mathrm{b}}$ Theoretical structures were obtained at B3LYP/6-311G $(d, p)$ for benzene, carbon monoxide, methyl, and benzoyl. $\operatorname{CASSCF}(9,10) / 6-31 \mathrm{G}(d)$ was used for the excited state $\left(T_{2}\right)$ of benzaldehyde, and $\operatorname{CASSCF}(9,10) / 6-311 \mathrm{G}(d, p)$ for the excited state $\left(T_{2}\right)$ of acetophenone.

${ }^{\mathrm{C}} C_{2 v}$ symmetry was imposed for the phenyl ring.

${ }^{\mathrm{d}}$ Dependent variables.

ring. In addition, bond distances and angles involving hydrogen atoms were fixed at values given by DFT.

For acetophenone, the molecular structure has been determined for the first time by UED. ${ }^{2}$ The combination of a high boiling point and reactivity in the apparatus made working with acetophenone a challenge. Moreover, the molecule, in principle, has numerous similar structural parameters which make the analysis nontrivial. The ratio pattern derived from the raw diffraction pattern is shown in Fig. 5.

The initial guess for the structural refinement was taken from the result of a DFT calculation at B3LYP/6$311 \mathrm{G}(d, p)$. As with benzaldehyde, the mean amplitudes of 


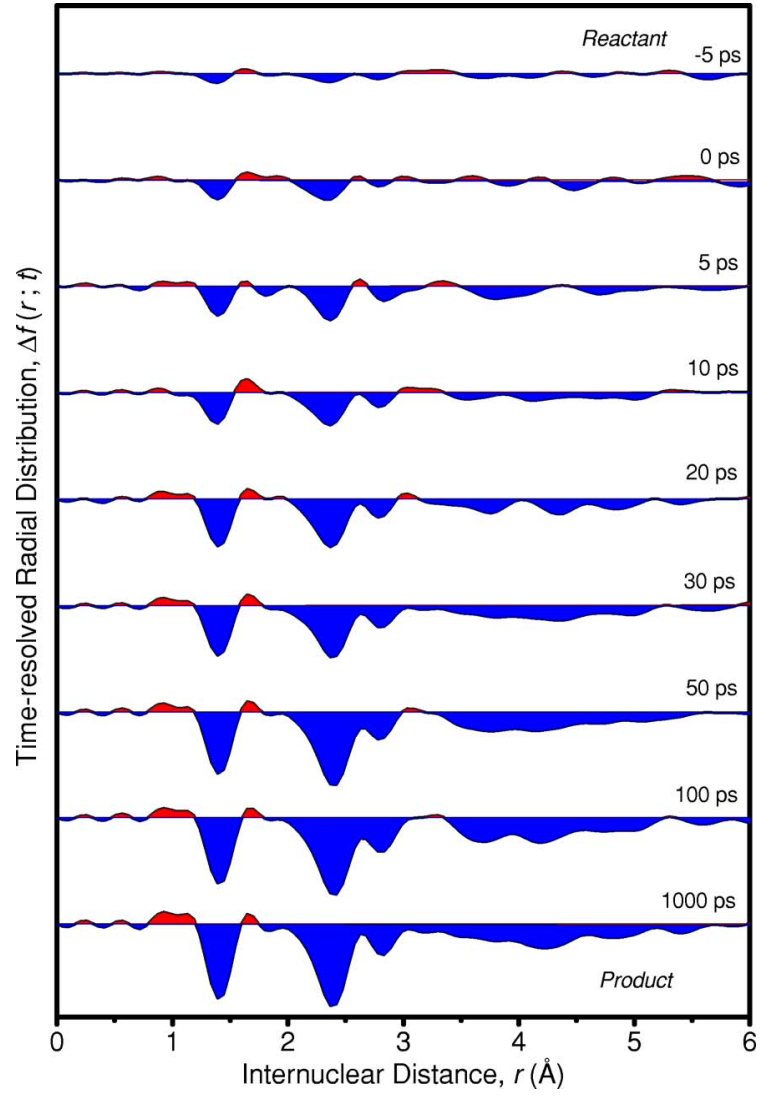

FIG. 9. The experimental $\Delta f(r ; t)$ for the photoinduced reaction of benzaldehyde at all time points. Note the rise and eventual leveling off of the difference signal.

vibration were obtained from empirical formulas ${ }^{68}$ and extrapolated to $483 \mathrm{~K}$ using the nozzle temperature as the temperature of the sample. The polynomial background and theory scaling factor were optimized for the best fit. The data range used for refinement was $s=3.5-14.6 \AA^{-1}$.

In order to simplify the refinement process, a $C_{2 v}$ symmetry was imposed for the aryl ring; however, the acetyl group was permitted unhindered rotation. The refined structural parameters of ground state acetophenone $(R=0.028)$ are listed in Table III. Bond lengths and angles agree with the theoretical values (from DFT) within $0.02 \AA$ and $2^{\circ}$, respectively. A relatively large discrepancy with DFT values was seen in the refined parameters related to the carbonyl and methyl groups: -0.017 and $+0.029 \AA$ for the $\mathrm{C}=\mathrm{O}$ and $\mathrm{C}-\mathrm{CH}_{3}$ distances, respectively, and $+3.5^{\circ}$ and $-2.8^{\circ}$ for the carbonyl and methyl angles with respect to the aryl skeleton. The orientation of the methyl hydrogen atoms is observed to deviate from the planar symmetric starting condition, reflecting the freedom of its torsional motion.

The data, $s M(s)$ and $f(r)$, are shown with the refined theoretical models in Fig. 6 for both benzaldehyde and acetophenone.

\section{B. Time-resolved structures: Benzaldehyde}

Figure 7 shows the two-dimensional frame-referenced patterns for benzaldehyde at $483 \mathrm{~K}$ obtained by subtracting the -100 ps ratio pattern from the ratio patterns of the other time points (see Sec. V D). The appearance of diffraction rings in the patterns signifies a structural change between the parent structure (at $-100 \mathrm{ps}$ ) and the structures formed as a result of the excitation pulse at time zero. The increase in magnitude of structural change with time is readily seen in the time-dependent data (Fig. 7). The same features are retained over time, but only increase in amplitude, signifying the growth in population of a common product structure throughout. The difference data point at $+50 \mathrm{ps}$ was selected for the refinement of the theoretical model on the basis of signal quality. The data from $s=3.1-13.9 \AA^{-1}$ were used, also owing to superior signal quality.

Calculations were performed to arrive at suitable starting geometries for structural refinement (see Sec. IV). Such trial models were based on the various molecules proposed in the literature as products of benzaldehyde photochemistry and the geometries of relevant excited states (see Sec. II). The actual products of photoexcitation were determined by comparing the +50 ps difference data with the theoretical models. Single product reaction models were compared with the data. The models that produce the lowest $R$ values are then used as the initial geometries for structural refinement. For benzaldehyde, the best product fits were the models corresponding to vibrationally "hot" ground state benzaldehyde $(R=0.668)$, benzene and carbon monoxide $(R=0.778)$, benzene triplet (quinoid and antiquinoid structures) and carbon monoxide $(R=0.448)$, and benzoyl radical and atomic hydrogen $(R=0.840)$. Structural refinement was attempted with each of these models individually, and in each case the result was an unphysical structure - two long bond distances $(>1.6 \AA)$ in the aryl ring. It was concluded that these models individually were each too near to undesired local minima and that a combination of them must be used to account for some more complicated photoreaction. Two-component model comparisons were then performed. Structural refinements were conducted with each dual model, and again, in each case two long bond distances occurred in the aryl rings, except in the cases where $T_{2}\left({ }^{*} \pi \pi^{*}\right)$ benzaldehyde is paired with either benzene and carbon monoxide or the phenyl and formyl radicals. Based on a better $\chi^{2}$ and previous experi-

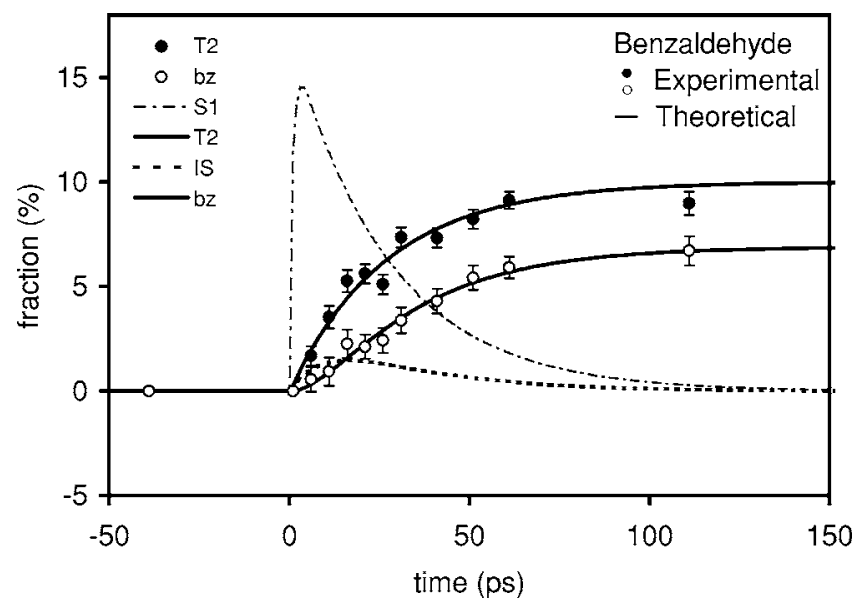

FIG. 10. The time-dependent relative fractions of the each of the benzaldehyde products, $T_{2}$ benzaldehyde (solid circles) and benzene + carbon monoxide (open circles). The physical and chemical products are fitted with a kinetic model yielding time constants of 42 and $61 \mathrm{ps}$, respectively. The time-dependent populations of the other relevant states are also shown. 

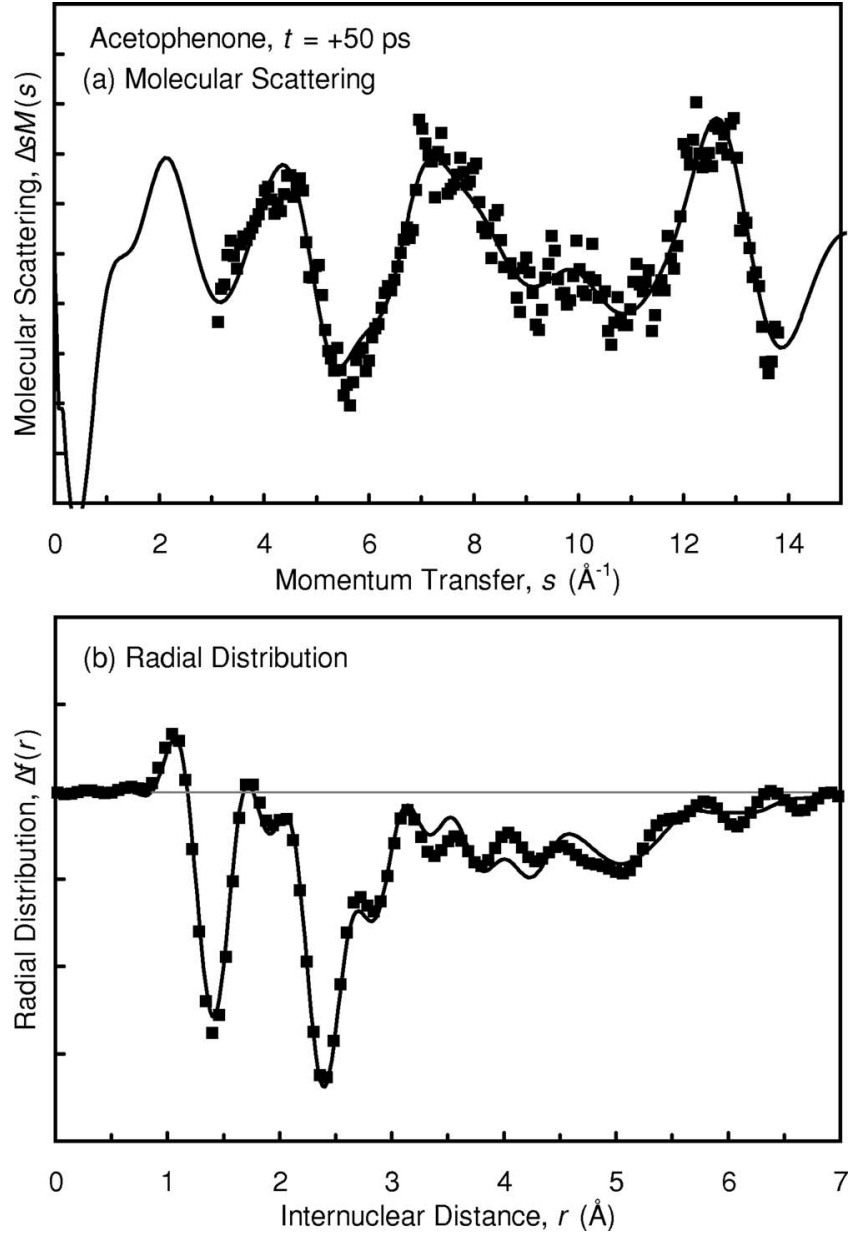

FIG. 11. Modified molecular scattering curves, $\Delta s M(s ; t)$, and difference radial distributions, $\Delta f(r ; t)$ for acetophenone at $t=+50$ ps upon $266.7 \mathrm{~nm}$ excitation. The experimental data points are shown as filled symbols and the refined theory is a solid line. $(R=0.473)$.

mental observations of benzene and carbon monoxide as photoproducts, ${ }^{11,12,35,37-39}$ the molecular mixture of benzene, carbon monoxide, and triplet $\pi \pi^{*}$ benzaldehyde were concluded to be the products of this reaction.

The final refined theoretical $s M(s)$ and $f(r)$ are shown with the data in Fig. $8(R=0.404)$. The structural parameters are listed in Table IV. A $C_{2 v}$ model was used for the quinoid benzaldehyde triplet; $a b$ initio calculations predict near- $C_{2 v}$ symmetry. Since preliminary refinements were rather insensitive to the carbonyl torsional angle, it was fixed at planarity for the final refinement. The structure of ground state benzene was refined with a $D_{6 h}$ model. The $\mathrm{C} \equiv \mathrm{O}$ distance of carbon monoxide and all structural parameters involving hydrogen atoms were fixed at $a b$ initio or DFT-derived values. Contrasting the results of the fit to the theoretically calculated structure shows a few deviations. Most notably, the double bonds of the quinoid ring are somewhat shorter than their theoretical counterparts $(1.322 / 1.322 \AA$ vs $1.362 / 1.358 \AA$, respectively) emphasizing the more electronlocalized, $\pi \pi^{*}$ character.

With the final structures now obtained, their contribution at each of the time points is evaluated via linear parameter fitting of the fractions and polynomial background terms. Figure 9 shows time-resolved experimental $\Delta f(r)$ for the re-
TABLE V. Relative product compositions.

\begin{tabular}{lrcc}
\hline \hline & & \multicolumn{2}{c}{ Fraction $(\%)$} \\
\cline { 3 - 4 } Molecule & Time $(\mathrm{ps})$ & Photophysical & Photochemical \\
\hline Benzaldehyde & -100 & 0 & 0 \\
& -50 & 0 & 0 \\
& -10 & 0 & 0 \\
-5 & $1.7 \pm 0.5$ & $0.6 \pm 0.6$ \\
& 0 & $3.5 \pm 0.5$ & $0.9 \pm 0.7$ \\
5 & $5.3 \pm 0.5$ & $2.3 \pm 0.7$ \\
& 10 & $5.6 \pm 0.5$ & $2.1 \pm 0.6$ \\
& 15 & $5.1 \pm 0.5$ & $2.4 \pm 0.6$ \\
& 20 & $7.4 \pm 0.5$ & $3.4 \pm 0.6$ \\
& 30 & $7.3 \pm 0.5$ & $4.3 \pm 0.6$ \\
& 40 & $8.2 \pm 0.5$ & $5.4 \pm 0.6$ \\
& 50 & $9.1 \pm 0.4$ & $5.9 \pm 0.5$ \\
& 100 & $9.0 \pm 0.6$ & $6.7 \pm 0.7$ \\
& 1000 & $10.9 \pm 0.5$ & $6.7 \pm 0.7$ \\
& -100 & 0 & 0 \\
50 & $8.9 \pm 0.8$ & $11.9 \pm 0.8$ \\
& 100 & $8.4 \pm 0.8$ & $16.8 \pm 0.8$ \\
\hline \hline
\end{tabular}

The error bars reported here are $3 \sigma$.

fined structures. The experimentally determined population change with time is shown in Fig. 10; relative product fractions at each time point are listed in Table V.

Both the photophysical $\left(T_{2}, \pi \pi^{*}\right.$ benzaldehyde $)$ and photochemical (benzene $+\mathrm{CO}$ ) channel products grow simultaneously. The apparent rise time constant of the channel forming $T_{2}$ benzaldehyde is $25 \pm 4 \mathrm{ps}$, determined by nonlinear fitting of a single step reaction. The rise time constant of the benzene-forming channel is somewhat longer, $38 \pm 5 \mathrm{ps}$. The final percent populations of $T_{2}$ benzaldehyde and benzene are $10.0 \% \pm 0.4 \%$ and $6.9 \% \pm 0.2 \%$, respectively, after reaching stationary state; the percentage is in reference to the total population of all species [Eq. (22)]. The nearly parallel behavior of the two product channels implies competitive bifurcation after origination from a single state, as no growth of one at the expense of the other was observed. At our excitation, to the $\pi \pi^{*} S_{2}$, it is known that the state has an ultrashort lifetime [ 250 fs (Ref. 20)]. Because of this, and the fact that intersystem crossing (ISC) is more efficient between states of different orbital excitations $\left(\pi \pi^{*} / n \pi^{*}\right)$, it follows that $T_{2}\left(\pi \pi^{*}\right)$ benzaldehyde must form from an $n \pi^{*}$ state via an efficient ISC, making $S_{1}$ benzaldehyde the origin state of bifurcation.

The bifurcation dynamic model of benzaldehyde can now be written as follows:

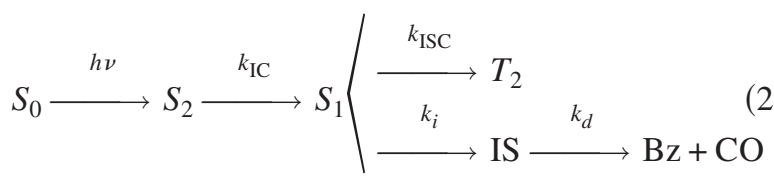

An intermediate structure (IS) is included in order to account for the difference in the rise time of the two channels bifurcated from the hot $S_{1} n \pi^{*}$ state. The presence of the IS is further supported by the electronic nature of $S_{1}$-dissociation from the $n \pi^{*}$ excited state correlates with the electronically excited state of $\mathrm{CO}$ which is not seen in the data. It follows 

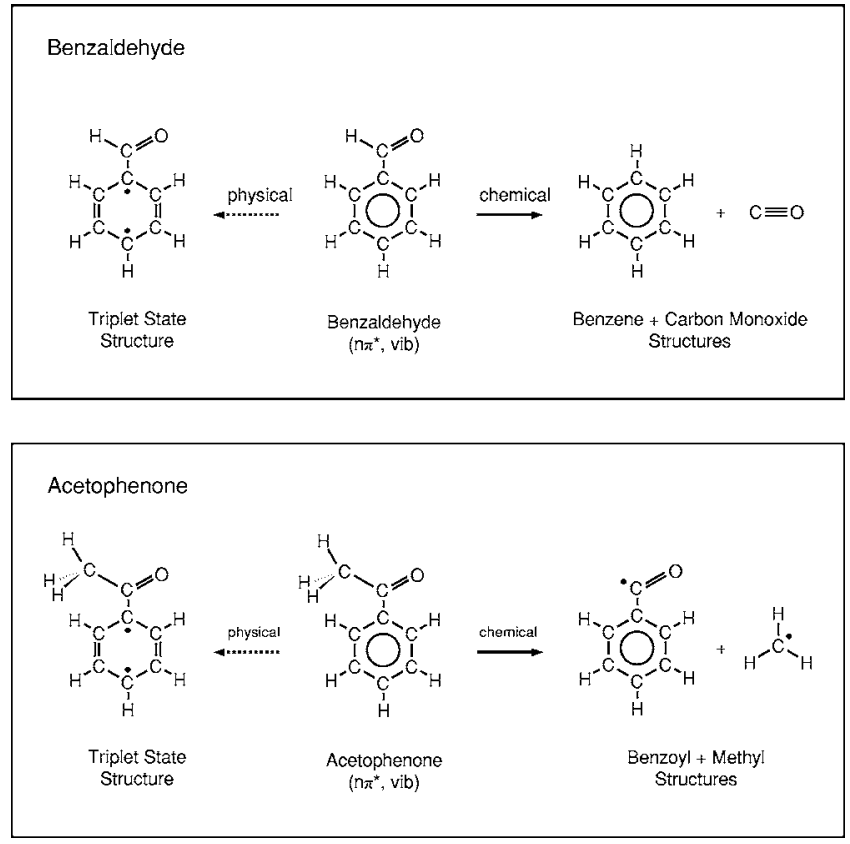

FIG. 12. Structures involved in the photophysical and photochemical processes of benzaldehyde (top) and acetophenone (bottom) on our experimental time scale.

that the total decay rate of the $S_{1}$ population is given by

$$
k=k_{\mathrm{ISC}}+k_{i}
$$

and the product branching ratio is

$$
\frac{P\left(T_{2}\right)}{P(\mathrm{Bz}+\mathrm{CO})}=\frac{k_{\mathrm{ISC}}}{k_{i}} \text {. }
$$

From the rise time of $25 \pm 4$ ps $\left(k=4.0 \times 10^{10} \pm 0.6\right.$ $\left.\times 10^{10} \mathrm{~s}^{-1}\right)$ for the faster channel and a branching ratio $\left(T_{2}: \mathrm{Bz}+\mathrm{CO}\right)$ of $10.0 \% \pm 0.4 \%: 6.9 \% \pm 0.2 \%$, the rate constants are found to be $k_{\mathrm{ISC}}=2.4 \times 10^{10} \mathrm{~s}^{-1} \quad\left(1 / k_{\mathrm{ISC}}=42 \mathrm{ps}\right)$ and $k_{i}=1.6 \times 10^{10} \mathrm{~s}^{-1} \quad\left(1 / k_{i}=61 \mathrm{ps}\right)$. A simulation of the population changes based on this bifurcation model is shown with the data in Fig. $10 ; k_{d}$ was taken to be $9.4 \times 10^{10} \mathrm{~s}^{-1}$ $\left(1 / k_{d}=11 \mathrm{ps}\right)$ from the initial rise of the benzene channel. The decay of the $S_{1}$ was not seen due to its structural similarity with the parent $S_{0}$; the very short-lived IS was not detectable because of its small population (see Fig. 10).

\section{Time-resolved structures: Acetophenone}

Diffraction data at three time points $(-100,+50$, and $+100 \mathrm{ps}$ ) were taken for acetophenone. The full temporal range of experimentation was limited because of inlet window coating mentioned in Sec. III C. The data from -100 ps were used as the reference and subtracted from the positive time points to generate the difference data. Perusal of both patterns and curves reveals a signal that is nearly, but not entirely, saturated 50 ps after the excitation laser pulse. Product analysis was conducted on both time points and found to yield equivalent results. The results presented hereafter were arrived at through analysis of the +50 ps difference data, unless otherwise noted.

Determination of the products of acetophenone photolysis was performed similar to that described previously for
Benzaldehyde

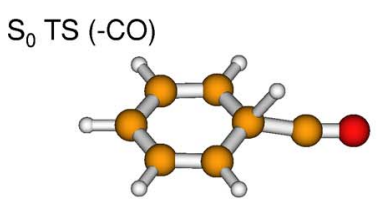

$n \pi^{*}$ TS (-CO)

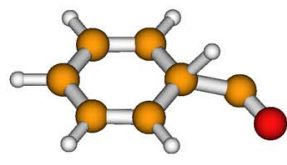

$n \pi^{*}$ IS $(-C O)$

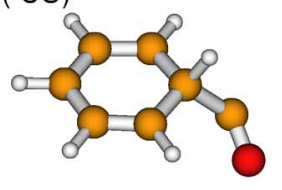

$n \pi^{\star}$ TS $(-H)$

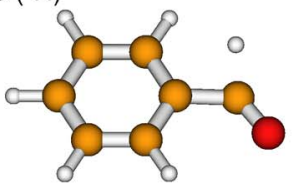

$\mathrm{n} \pi^{*}$ TS $\left(-\mathrm{C}_{6} \mathrm{H}_{5}\right)$
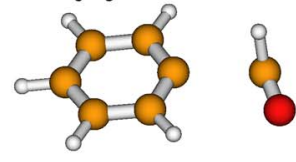

Acetophenone

$\mathrm{S}_{0} \mathrm{TS}(-\mathrm{CO})$

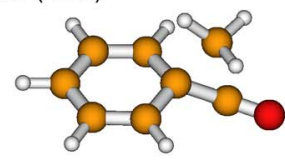

$n \pi^{*}$ TS (-CO)

$\mathrm{n} \pi^{\star}$ IS (-CO)

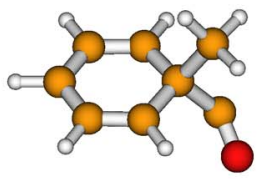

$n \pi^{\star} \mathrm{TS}\left(-\mathrm{CH}_{3}\right)$

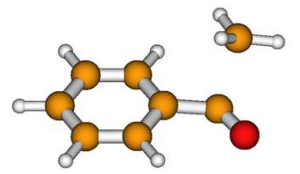

$\mathrm{n} \pi^{*} \mathrm{TS}\left(-\mathrm{C}_{6} \mathrm{H}_{5}\right)$

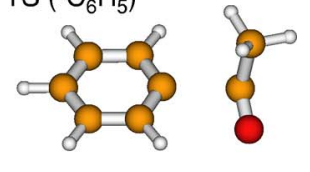

FIG. 13. The geometries of transition states and intermediates in the reactions of benzaldehyde (left) and acetophenone (right). Calculations were performed at the B3LYP/6-311G $(d, p)$ level and include pathways observed and some that represent other possibilities.

benzaldehyde. First, structures proposed in the literature and our own calculations were compared with the difference data. Products used in these comparisons have been speculated by previous photochemical and photophysical works. The best single product fit, by $R$ value, is the channel leading to toluene and carbon monoxide as products $(R=0.636)$. Attempts at structural refinement of the single products produced geometries that were unphysical (direct bond distances $>1.6 \AA$ ). The best dual product fit is the product combination of $T_{2}\left(\pi \pi^{*}\right)$ acetophenone, toluene, and carbon monoxide $(R=0.584)$.

Continuing to follow the procedure used for analyzing the benzaldehyde data, structural refinement was also performed on several of the dual product models. The molecular dissociation pathway which had worked well for benzaldehyde was not compatible with the acetophenone data despite the favorable $a b$ initio comparison. Refinement of that model caused the contribution of $T_{2}$ acetophenone to be eliminated entirely leaving only toluene and carbon monoxide. The structure of toluene was also changed significantly; two of the $\mathrm{C}-\mathrm{C}$ bonds of the aryl ring stretched to $1.57 \AA$ and the methyl group became separated from the ring by $1.98 \AA$. The improbable geometry excluded this product channel from 
TABLE VI. Statistical and mode-limited rate constants.

\begin{tabular}{|c|c|c|c|c|c|c|c|c|c|}
\hline \multirow[b]{2}{*}{ Molecule } & \multirow{2}{*}{\multicolumn{2}{|c|}{ Channel }} & \multicolumn{4}{|c|}{ Energy $^{\mathrm{a}, \mathrm{b}}\left(\mathrm{cm}^{-1}\right)$} & \multicolumn{3}{|c|}{$\operatorname{Rate}^{\mathrm{c}, \mathrm{d}}\left(\mathrm{s}^{-1}\right)$} \\
\hline & & & Reactant & TS & IS & Product & $k_{6}$ & $k_{n}$ & $k_{s}$ \\
\hline \multirow[t]{6}{*}{ Benzaldehyde } & \multirow[t]{3}{*}{$S_{0}$} & $-\mathrm{CO}$ & 0 & 32218 & $\cdots$ & 460 & $1.7 \times 10^{10}$ & $2.1 \times 10^{8}$ & $5.4 \times 10^{0}$ \\
\hline & & $-\mathrm{H}$ & 0 & $\cdots$ & $\cdots$ & 32679 & & & \\
\hline & & $-\mathrm{C}_{6} \mathrm{H}_{5}$ & 0 & $\cdots$ & $\cdots$ & 34611 & & & \\
\hline & \multirow[t]{3}{*}{$S_{1}$} & $-\mathrm{CO}$ & 26921 & 35474 & 28439 & 30087 & $1.6 \times 10^{11}$ & $1.3 \times 10^{10}$ & $1.0 \times 10^{7}$ \\
\hline & & $-\mathrm{H}$ & 26921 & 36297 & $\ldots$ & 32682 & $7.7 \times 10^{10}$ & $5.6 \times 10^{9}$ & $6.6 \times 10^{6}$ \\
\hline & & $-\mathrm{C}_{6} \mathrm{H}_{5}$ & 26921 & 36772 & $\cdots$ & 34611 & $5.0 \times 10^{10}$ & $3.9 \times 10^{9}$ & $1.6 \times 10^{7}$ \\
\hline \multirow[t]{6}{*}{ Acetophenone } & \multirow[t]{3}{*}{$S_{0}$} & $-\mathrm{CO}$ & 0 & 33697 & $\cdots$ & 1816 & $6.2 \times 10^{9}$ & $1.4 \times 10^{5}$ & $8.0 \times 10^{-3}$ \\
\hline & & $-\mathrm{CH}_{3}$ & 0 & $\cdots$ & $\cdots$ & 29751 & & & \\
\hline & & $-\mathrm{C}_{6} \mathrm{H}_{5}$ & 0 & $\cdots$ & $\cdots$ & 34291 & & & \\
\hline & \multirow[t]{3}{*}{$S_{1}$} & $-\mathrm{CO}$ & 27279 & $\times$ & 29810 & 30890 & & & \\
\hline & & $-\mathrm{CH}_{3}$ & 27279 & 34257 & $\ldots$ & 29751 & $6.7 \times 10^{11}$ & $1.6 \times 10^{10}$ & $3.5 \times 10^{8}$ \\
\hline & & $-\mathrm{C}_{6} \mathrm{H}_{5}$ & 27279 & 36951 & $\cdots$ & 34291 & $3.5 \times 10^{10}$ & $2.1 \times 10^{8}$ & $2.9 \times 10^{6}$ \\
\hline
\end{tabular}

The energy corrections for $S_{1}\left({ }^{1} n \pi^{*}\right)$ reactions are 3906 and $3710 \mathrm{~cm}^{-1}$ for benzaldehyde and acetophenone, respectively (see text).

${ }^{b}$ The energy corrections for products are $2276 \mathrm{~cm}^{-1}$ for $\mathrm{C}_{6} \mathrm{H}_{5} \mathrm{CO}+\mathrm{H}$ and $3091 \mathrm{~cm}^{-1}$ for $\mathrm{C}_{6} \mathrm{H}_{5} \mathrm{CO}+\mathrm{CH}_{3}$.

${ }^{\mathrm{c}} k_{6}, k_{n}$, and $k_{s}$ denote statistical rate constants for various models of energy coupling among phenyl, methyl, and carbonyl groups. $k_{6}$ (for six involved modes) is obtained by assuming that the phenyl and methyl groups do not participate in energy randomization (spectator model), and $k_{s}$ by assuming full randomization of reactant energy amongst all vibrational modes. $k_{n}$ is obtained by assuming partial randomization where only $n$ vibrational modes participate in the dissociation; $n=10$ for benzaldehyde and $n=16$ for acetophenone are used.

${ }^{\mathrm{d}}$ Thermal energies at $500 \mathrm{~K}$ were added to the excitation energy of $37500 \mathrm{~cm}^{-1}(266.7 \mathrm{~nm})$.

further consideration. Structural refinement of several other two-product models resulted in similar behavior.

Well-behaved structural refinement was found with the model corresponding to the channels forming $T_{2}$ acetophenone and the benzoyl and methyl radicals. The final refined theoretical model is shown with the data in Fig. 11. The fitted structural parameters are listed in Table IV along with those predicted by theory. In this refinement it was necessary to employ structural dependencies to simplify the fitting. In addition to the restriction of $C_{2 v}$ symmetry for the aryl rings in both benzoyl radical and $T_{2}$ acetophenone, the models of both molecules were further restricted. Only one distance and one angle were refined in the ring of the benzoyl radical and, for $T_{2}$ acetophenone, all single bonds were defined by one "fittable" parameter. Furthermore, the skeletal angles of the $T_{2}$ acetophenone ring were defined as one, as were the angles of the carbonyl moiety. And, as is typical of all ring structures, some parameters were unable to be defined and simply drifted with the changes of the other, fittable parameters. The torsional angles for carbonyl groups in the benzoyl radical and in $T_{2}$ acetophenone were fixed in the plane of the ring, as indicated by theory and preliminary fitting results. Differences between the parameters derived from the diffraction data and those calculated by theory were less than $0.03 \AA$ for bond distances and $3^{\circ}$ for angles, or were within the error bar. The double and single bonds of the $T_{2}$ acetophenone ring are clearly resolved demonstrating the loss of aromaticity and its $\pi \pi^{*}$ nature.

Upon the acquisition of the structures of the reaction products, their fractional contributions were obtained at the +100 ps difference time point (recall that structural refinement was conducted at the $+50 \mathrm{ps}$ difference time point). From +50 to +100 ps the fractional amount of $T_{2}$ acetophenone remains unchanged, but the amount of fragmentation into benzoyl and methyl radicals increases over the two time points from $11.9 \% \pm 0.8 \%$ at +50 ps to $16.8 \% \pm 0.8 \%$ at $+100 \mathrm{ps}$. This is a similar case to that reported above for benzaldehyde where dissociation also occurs at a slower rate. Because of the limited data points we cannot elucidate the exact nature of the bifurcation. However, the structures determined lead us, in analogy with benzaldehyde, to the following mechanism:

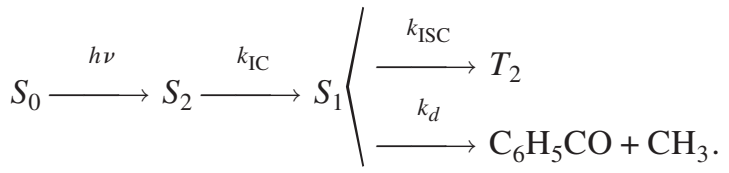

The relative fractions of the products are listed in Table V. The observed structures involved in the photophysical and photochemical bifurcations in both benzaldehyde and acetophenone are shown in Fig. 12.

\section{DISCUSSION}

The results of our UED studies of benzaldehyde and acetophenone can be summarized as follows. The ground state structures of benzaldehyde and acetophenone were determined. The structural parameters refined for the benzaldehyde data agree well with previous conventional electron diffraction results. ${ }^{33}$ To our knowledge, the molecular structure of acetophenone has not been determined previously. For both systems, it was possible to study the structural dynamics following ultrafast laser excitation at $266.7 \mathrm{~nm}$ to the $S_{2}\left(\pi \pi^{*}\right)$ excited state. The photophysical and photochemical channels were observed in competition and the resultant molecular structures characterized. Time scales were also determined. 

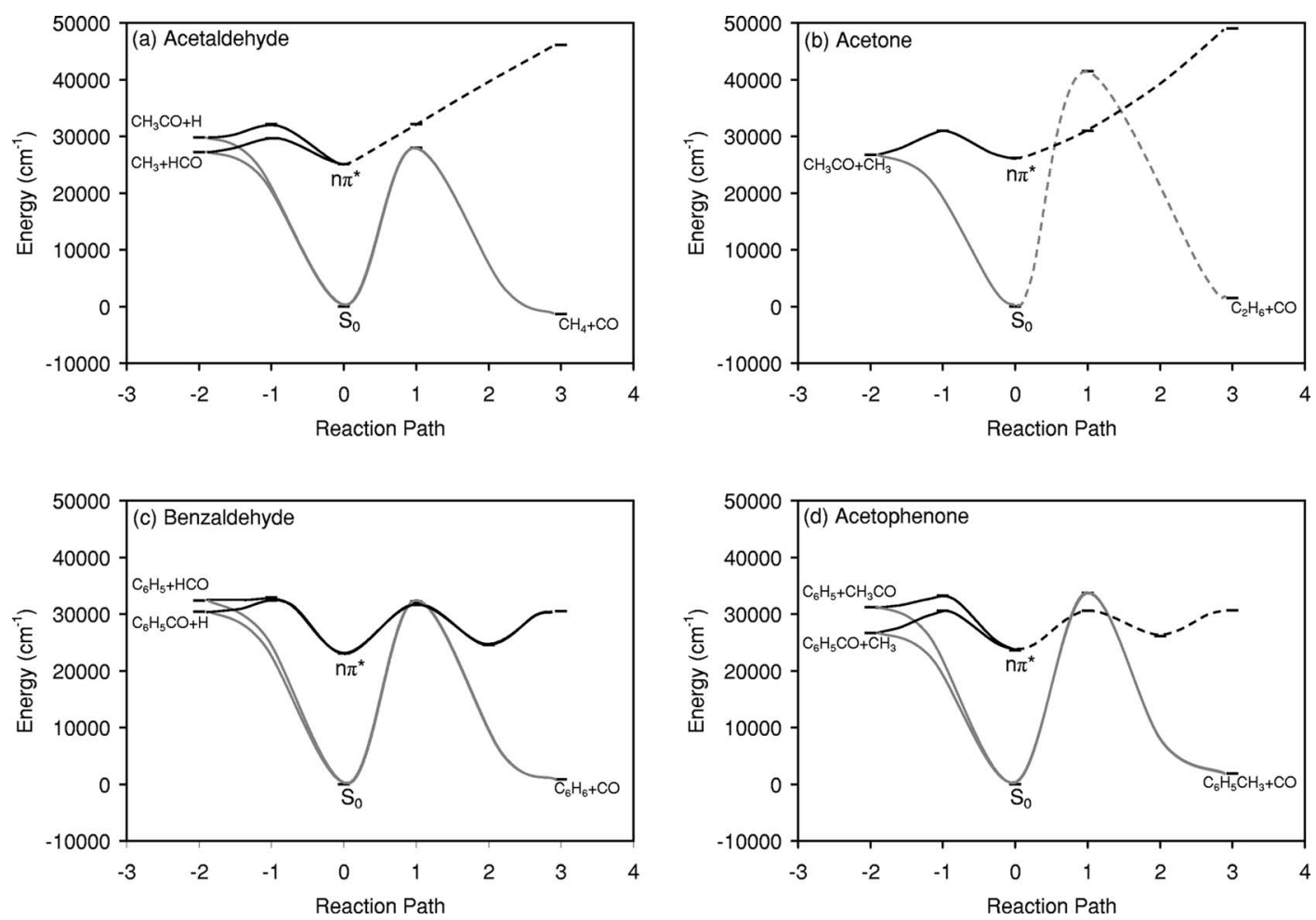

FIG. 14. Calculated reaction coordinates of radical cleavage and molecular dissociation pathways of (a) acetaldehyde, (b) acetone, (c) benzaldehyde, and (d) acetophenone on the ground electronic surface and the $T_{1}, n \pi^{*}$ surface. Calculations were performed at the B3LYP/6-311G $(d, p)$ level.

The refinement of the structures present in the timeresolved diffraction data for acetophenone and benzaldehyde results from the bifurcation on the excited $S_{1} n \pi^{*}$ state, following femtosecond internal conversion from $S_{2}$, which results in the formation of a quinoid triplet structure and the products of dissociation. For benzaldehyde the dissociation products are the closed-shell molecular species, benzene and carbon monoxide. For acetophenone they are the benzoyl and methyl radicals.

It is to be noted that UED is uniquely suited for elucidating such excited state behavior. The $T_{2}$ states of these molecules are "dark" and have eluded spectroscopic studies for decades. UED does not rely on brightness and instead reveals information regarding every structure present in the interaction region at any given time.

\section{A. Structural dynamics}

Given the UED results above, the following dynamical picture of aromatic carbonyl photophysics and photochemistry can be drawn. Excitation at $266.7 \mathrm{~nm}$ promotes population of both acetophenone and benzaldehyde into the $\pi \pi^{*} S_{2}$ state which decays rapidly by internal conversion into $S_{1}$. For benzaldehyde at our excitation, the decay time is roughly $250 \mathrm{fs}$ and for acetophenone $S_{2}$ lives less than $140 \mathrm{fs}{ }^{20}$ In the nascent vibrational levels of $S_{1}$, aromatic carbonyl molecules experience a bifurcation of pathways. A physical pathway moves some population into the triplet manifold, the $\pi \pi^{*} T_{2}$ state, and a competitive chemical pathway causes dissociation into products.
For benzaldehyde, the physical channel (ISC) from $S_{1}$ proceeds with a rate of $2.4 \times 10^{10} \mathrm{~s}^{-1}\left(1 / k_{\mathrm{ISC}}=42 \mathrm{ps}\right)$ and survives up to at least $1 \mathrm{~ns}$; the measured rate is $k_{\mathrm{ISC}}+k_{i}$ [see Eq. (29)]. The triplet structure was identified as that of the $\pi \pi^{*} T_{2}$ and, compared to the ground state, the phenyl ring is seen to distort as aromaticity is lost- - two double bonds and four single bonds make up the quinoid ring. Along the chemical pathway, the benzene and carbon monoxide products are formed stepwise, involving an IS, as determined by the observed kinetics detailed in Sec. VI B.

After internal conversion to the $n \pi^{*} S_{1}$, excitation is localized mainly on the $\mathrm{C}=\mathrm{O}$ bond of the carbonyl group. According to our quantum chemical calculations, the carbonyl group remains coplanar with the phenyl ring; however, the $\mathrm{H}-\mathrm{C}=\mathrm{O}$ out-of-plane bending mode is reduced in frequency $\left(642 \mathrm{~cm}^{-1}\right.$ on $n \pi^{*}$ compared with $1033 \mathrm{~cm}^{-1}$ on $\left.S_{0}\right)$. The more facile large-amplitude motion of the aldehydic hydrogen atom allows for its capture by $\mathrm{C} 1$ and the subsequent formation of a transition state (TS) where the $\mathrm{H}$ is shared by $\mathrm{C} 1$ and C7 (shown in Fig. 13). This TS connects the $n \pi^{*}$ state to IS where $\mathrm{C} 1$ is $s p^{3}$ hybridized and bound to both $\mathrm{H}$ and $\mathrm{CO}$ (also shown in Fig. 13). The aldehydic hydrogen atom and the $\mathrm{CO}$ moiety are loosely bound and distorted from the molecular plane and either can be lost from IS by simple cleavage. Pathways are depicted in Fig. 14. Perturbation present in the $\mathrm{C}=\mathrm{O}$ moiety of the $n \pi^{*}$ state switches in the intermediate to a $\pi \pi^{*}$-like perturbation of the phenyl group resulting in a "pseudoprefulvenic" six-membered ring structure. The $\mathrm{C}=\mathrm{O}$ distance in the intermediate is identical to the $\mathrm{C}=\mathrm{O}$ distance of the acetyl radical ground state $(1.180 \AA)$, indicating the stability of the CO moiety. 

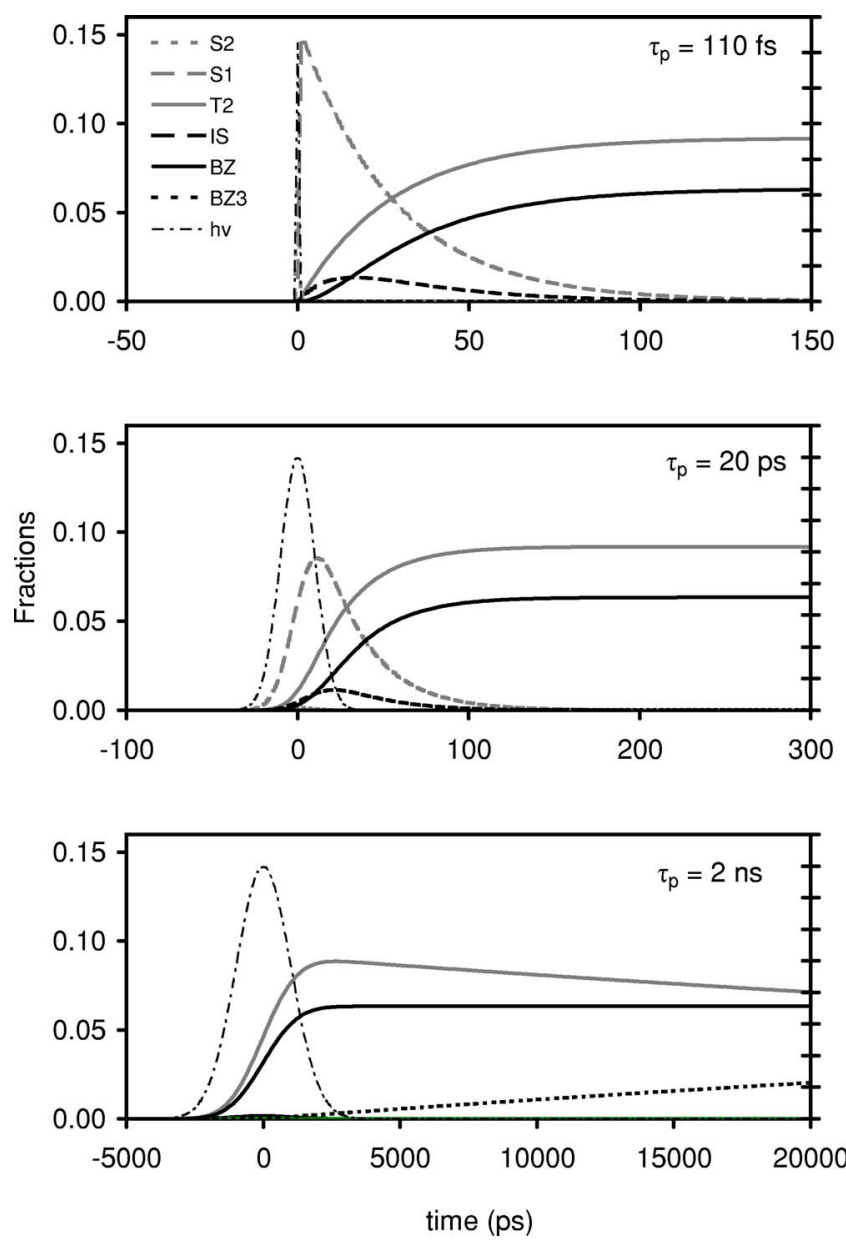

FIG. 15. Dynamical model of population flow of states relevant to the photophysical and photochemical processes of excited benzaldehyde. The panels show the relative component fractions based on laser excitation by a $266.7 \mathrm{~nm}$ pulse of (a) $110 \mathrm{fs}$, (b) $20 \mathrm{ps}$, or (c) $2 \mathrm{~ns}$ duration.

UED results show that the rearrangement step ( $S_{1}$ to IS) takes place at a rate of $1.6 \times 10^{10} \mathrm{~s}^{-1}\left(1 / k_{i}=61 \mathrm{ps}\right)$; the measured rate is $k_{\mathrm{ISC}}+k_{i}$ [see Eq. (29)]. But, statistical RiceRamsperger Kassel-Marcus (RRKM) ${ }^{75}$ calculations predict a rate several orders of magnitude slower (see Table VI), implying that energy redistribution among vibrational modes may be incomplete. The TS between the $n \pi^{*}$ state and IS is lower in energy than both the $n \pi^{*}$ TS leading to benzoyl and hydrogen radicals as well as an $S_{0}$ TS leading directly to benzene and carbon monoxide products. The structures of the various theoretical transition states and intermediates are shown in Fig. 13. Passage from the excited state IS to the products may proceed in different ways. The most direct route would be dissociation of $\mathrm{CO}$ from IS. However, an alternative route exists through the ground state-although a frequency calculation confirms the stability of IS in the excited state, it immediately undergoes CO loss when put on the $S_{0}$ surface. Internal conversion of IS followed by instantaneous dissociation could be the more likely path. The threshold energy for benzene formation from benzaldehyde is known to be just below the $S_{2}$ origin with which the above theoretical estimation agrees quite reasonably., ${ }^{5,7,16}$ If instead we were to assume that the molecular dissociation occurs after internal conversion from $S_{1}$ to $S_{0}$, we have calcu-
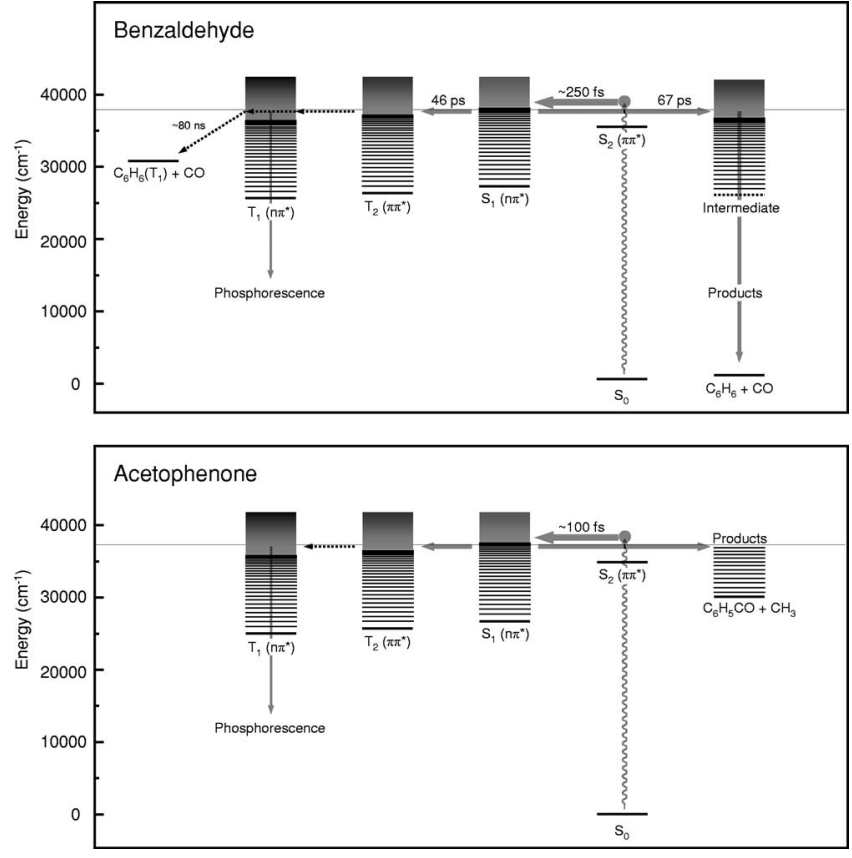

FIG. 16. The bifurcation into photochemical and photophysical processes upon excitation of benzaldehyde (top) and acetophenone (bottom).

lated that the large barrier height $\left(32218 \mathrm{~cm}^{-1}\right)$ will render the reaction much slower than the time scale observed (see Table VI).

For acetophenone, the bifurcation from $S_{1}$ is also seen as a competition between chemical and physical pathways. The physical pathway results in the formation of a $\pi \pi^{*}$ triplet, structurally similar to the quinoid ring seen for benzaldehyde. However, the chemical result is quite different. On the excited state, the methyl group does not show the same propensity for large-amplitude motion as was seen for the aldehydic hydrogen of $S_{1}$ benzaldehyde and its capture by the phenyl ring does not occur. In other words, a pathway (or TS) could not be found for the molecular dissociation channel from the $n \pi^{*}$ state-although an IS such as that found for the benzaldehyde dissociation can be calculated for acetophenone (see Fig. 13), no direct trajectory exists to link it to $S_{1}$. Methyl loss is the lowest energy chemical channel found from the $n \pi^{*}$ surface. The quantum chemical calculations above agree well with our experimental observations; molecular dissociation for benzaldehyde and radical cleavage for acetophenone are the favored chemical pathways from their respective $n \pi^{*}$ states.

\section{B. Spectroscopic observations revisited}

The $T_{2}$ states of acetophenone and benzaldehyde are electronic states that have eluded direct characterization by all previous experimental methods. As described in Sec. II, phosphorescence studies have supplied all that is known of the state and its relationship to the bright $T_{1}$. Some previous researchers ${ }^{11,38}$ employed a kinetic model with an intermediate state in the triplet manifold, with a lifetime long enough to explain the collision-induced quenching of chemistry and the enhancement of phosphorescence in high pressure envi- 
ronments. The UED results permit us to identify this reactive, yet quenchable, intermediate as $T_{2}$. If $T_{2}$ is allowed to evolve in the absence of collisions, dissociation occurs to produce the triplet benzene observed by other researchers on longer time scales. ${ }^{38,39}$ The dissociation time constant for this reaction would be about $80 \mathrm{~ns}$ at our excitation energy. The triplet state of $\pi \pi^{*}$ benzene and ground state of $\mathrm{CO}$ theoretically correlate as molecular dissociation products of $T_{2} \pi \pi^{*}$ benzaldehyde. If, however, $T_{2}$ loses its excess vibrational energy through collisions, the only significant relaxation pathway becomes phosphorescent emission through $T_{1}$. In other words, phosphorescence is the dominant process in aromatic carbonyl compounds possessing little excess energy above the $S_{1}$ origin. In the isolated molecule, at energies above $S_{2}$ excitation the expected yield of benzene and carbon monoxide is nearly unity (phosphorescence is a minor channel) ${ }^{4,11,37}$ This unity yield of benzene is actually the sum of two separate benzene-forming reactions: the fast singlet channel and the slow triplet channel.

The steady population of $T_{2}$ up to $1 \mathrm{~ns}$ delay seen by UED confirms the slowness of a further chemical channel but also signifies the lack of irreversible population transfer to $T_{1}$. Since the energy gap between $T_{2}$ and $T_{1}$ is known to be so small, it may be explained by a mixed state or by rapid and "reversible" internal conversion between the two. However, the structural similarity of $T_{1}$ and $S_{0}$ precludes the detection of $T_{1}$ in this UED study, and confirmation of its presence was not possible.

Benzene formation through the photodissociation of benzaldehyde has previously been the subject of multiphoton ionization (MPI) studies using different pulse widths. ${ }^{35-37,39}$ These inquiries have provided a reaction time scale between $20 \mathrm{ps}$ and $2 \mathrm{~ns}$. In order to unite our UED results with these from the literature, simulations of population evolution have been performed following the photophysical and photochemical mechanisms described in Eq. (28) and using the rate constants available. Population changes were calculated by numerical integration of the differential equations of the kinetic model using the fourth order Runge-Kutta method. ${ }^{72}$ Multiphoton ionization of neutral species to their ionic counterparts was also included. Laser pulse widths of $110 \mathrm{fs}$, $20 \mathrm{ps}$, and $2 \mathrm{~ns}$ are used to model each type of experiment (ours and others, see Refs. 35-37). The time-dependent population changes and laser profiles are shown in Fig. 15. Using a $20 \mathrm{ps}$ ultraviolet pulse, the simulation predicts a branching ratio of 94\%: 6\% for molecular and benzene ion yields, respectively, while one for a 2 ns excitation laser pulse predicts a ratio of 6\%: $94 \%$. Both results are in qualitative agreement with the MPI experiments. ${ }^{35}$ It should be noted that our simulation is based on the assumption that two-photon absorption cross sections for photoionizations of both excited benzaldehyde and ground state benzene are similar, although in reality they should strongly depend on wavelength, electronic structure, and resonance.

To better understand the differences between the photochemistries of acetophenone and benzaldehyde, it is valuable to compare them with the aliphatic carbonyl molecules, acetone and acetaldehyde (see Fig. 14). Acetaldehyde is a prototypical aldehyde and is known to undergo "molecular dis- sociation" (CO abstraction $)^{76}$ as well as "homolytic cleavage" (radical fragmentation) upon UV absorption. Acetone, the prototypical ketone, however, undergoes only a homolytic radical cleavage well known as the Norrish type-I reaction. ${ }^{65,77}$ A molecular dissociation channel has never been reported. It has been established that the molecular dissociation pathway of acetaldehyde occurs after internal conversion to the hot $S_{0}$ while radical channels take place after ISC. Radical cleavage of acetone is also believed to occur after ISC to the triplet manifold; however, it has been recently proposed ${ }^{65}$ and confirmed ${ }^{78}$ that fragmentation occurs directly from $S_{1}$ and yields an electronically excited acetyl radical which then undergoes internal conversion.

Although there are some analogies between aromatic and aliphatic carbonyl compounds, the physics and chemistry of aromatic carbonyl molecules are more complex due to the addition of close-lying $\pi \pi^{*}$ states to the manifold of low-lying $n \pi^{*}$ states provided by the carbonyl group. Namely, an $n \pi^{*}$ state, when located near a $\pi \pi^{*}$ state, can provide additional pathways for an aromatic carbonyl via energetically stable intermediates. Therefore, benzaldehyde (aromatic) may perform the molecular dissociation reaction through an intermediate structure on the excited state where it is the most energetically favored channel, while acetaldehyde (aliphatic) undergoes molecular dissociation on its ground state. Acetophenone, lacking a mobile hydrogen, may very well fragment following the mechanism of its aliphatic analog, acetone.

\section{CONCLUSION}

Structural dynamics of the aromatic carbonyls benzaldehyde and acetophenone (see Fig. 16) have been studied using the methodology of ultrafast electron diffraction. Following $S_{2}\left(\pi \pi^{*}\right)$ excitation at $266.7 \mathrm{~nm}$, a bifurcation of pathways between photophysics (intersystem crossing) and photochemistry (molecular dissociation or radical cleavage) was observed. Intersystem crossing in both molecules results in a "quinoidlike" structure for the excited state, $T_{2}\left(\pi \pi^{*}\right)$. The product structures revealed different chemistries for benzaldehyde and acetophenone, elucidating the fundamental disparity between aromatic aldehydes and ketones. Theoretical calculations support that the distinction results from a more facile large-amplitude motion of the aldehydic hydrogen compared to the methyl substituent. The photophysics of these molecules is made clear as UED has allowed for the direct detection and structural characterization of the dark structure of the $T_{2}$ state. Furthermore, the relationship between physical and chemical pathways in these molecules has been elucidated and a comprehensive picture of the structure of excited-state landscapes and pathways has been drawn.

\section{ACKNOWLEDGMENTS}

We would like to thank the National Science Foundation and the Air Force Office of Scientific Research for providing support for this work. We would also like to thank Dr. Shoujun $\mathrm{Xu}$ for his assistance in the design of the sample inlet. 
${ }^{1}$ R. Srinivasan, J. S. Feenstra, S. T. Park, S. Xu, and A. H. Zewail, Science 307, 558 (2005).

${ }^{2}$ J. S. Feenstra, S. T. Park, and A. H. Zewail, J. Chem. Phys. 123, 221104 (2005).

${ }^{3}$ G. Thiault, A. Mellouki, G. Le Bras, A. Chakir, N. Sokolowski-Gomez, and D. Daumont, J. Photochem. Photobiol., A 162, 273 (2004).

${ }^{4}$ Y. Hirata and E. C. Lim, J. Chem. Phys. 72, 5505 (1980).

${ }^{5}$ T. Itoh, Chem. Phys. Lett. 133, 254 (1987).

${ }^{6}$ M. B. Robin and N. A. Kuebler, J. Am. Chem. Soc. 97, 4822 (1975).

${ }^{7}$ T. Itoh, H. Baba, and T. Takemura, Bull. Chem. Soc. Jpn. 51, 2841 (1978).

${ }^{8}$ N. Ohmori, T. Suzuki, and M. Ito, J. Phys. Chem. 92, 1086 (1988).

${ }^{9}$ E. Villa, A. Amirav, W. Chen, and E. C. Lim, Chem. Phys. Lett. 147, 43 (1988).

${ }^{10}$ A. Inoue and N. Ebara, Chem. Phys. Lett. 109, 27 (1984).

${ }^{11}$ M. Berger, I. L. Goldblatt, and C. Steel, J. Am. Chem. Soc. 95, 1717 (1973).

${ }^{12}$ U. Brühlmann, M. Monella, P. Russegger, and J. R. Huber, Chem. Phys. 81, 439 (1983).

${ }^{13}$ C. R. Silva and J. P. Reilley, J. Phys. Chem. 100, 17111 (1996).

${ }^{14}$ M. Biron and P. Longin, Chem. Phys. Lett. 116, 250 (1985).

${ }^{15}$ T. Itoh, Chem. Phys. Lett. 151, 166 (1988).

${ }^{16}$ T. Itoh, T. Takemura, and H. Baba, Chem. Phys. Lett. 40, 481 (1976).

${ }^{17}$ M. Kiritani, T. Yoshii, N. Hirota, and M. Baba, J. Phys. Chem. 98, 11265 (1994).

${ }^{18}$ M. Koyanagi and L. Goodman, Chem. Phys. 39, 237 (1979).

${ }^{19}$ O. Sneh and O. Cheshnovsky, J. Phys. Chem. 95, 7154 (1991).

${ }^{20}$ S.-H. Lee, K.-C. Tang, I.-C. Chen, M. Schmitt, J. P. Shaffer, T. Schultz, J. G. Underwood, M. Z. Zgierski, and A. Stolow, J. Phys. Chem. A 106, 8979 (2002)

${ }^{21}$ Y. Kanda, H. Kaseda, and T. Matamura, Spectrochim. Acta 20, 1387 (1964).

${ }^{22}$ H. Murai and K. Obi, J. Phys. Chem. 79, 2446 (1975).

${ }^{23}$ S. Nagaoka and N. Hirota, Bull. Chem. Soc. Jpn. 56, 3381 (1983); L. Goodman and M. Koyanagi, Mol. Photochem. 4, 369 (1972).

${ }^{24}$ I. Ozkan and L. Goodman, Chem. Phys. Lett. 64, 32 (1979).

${ }^{25}$ H. Hayashi and S. Nagakura, Mol. Phys. 27, 969 (1974).

${ }^{26}$ D. G. Leopold, R. J. Hemley, and V. Vaida, J. Chem. Phys. 75, 4758 (1981).

${ }^{27}$ L. Goodman and I. Ozkan, Chem. Phys. Lett. 61, 216 (1979).

${ }^{28}$ C. Mijoule and P. Yvan, Chem. Phys. Lett. 43, 524 (1976); J. E. Ridley and M. C. Zerner, J. Mol. Spectrosc. 76, 71 (1979).

${ }^{29}$ V. Molina and M. Merchán, J. Phys. Chem. A 105, 3745 (2001).

${ }^{30}$ W.-H. Fang and D. L. Phillips, ChemPhysChem 3, 889 (2002).

${ }^{31}$ Y.-W. Wang, H.-Y. He, and W.-H. Fang, J. Mol. Struct.: THEOCHEM 634, 281 (2003).

${ }^{32}$ R. K. Kakar, E. A. Rinehart, C. R. Quade, and T. Kojima, J. Chem. Phys. 52, 3803 (1970).

${ }^{33}$ K. B. Borisenko, C. W. Bock, and I. Hargittai, J. Phys. Chem. 100, 7426 (1996)

${ }^{34}$ V. S. Antonov, V. S. Letokhov, and A. N. Shibanov, Appl. Phys. 22, 293 (1980).

${ }^{35}$ S. R. Long, J. T. Meek, P. J. Harrington, and J. P. Reilley, J. Chem. Phys. 78, 3341 (1983).

${ }^{36}$ J. J. Yang, D. A. Gobeli, and M. A. El-Sayed, J. Phys. Chem. 89, 3426 (1985).

${ }^{37}$ J. J. Yang, D. A. Gobeli, R. S. Pandolfi, and M. A. El-Sayed, J. Phys. Chem. 87, 2255 (1983)

${ }^{38}$ A. V. Polevoi, V. M. Matyuk, G. A. Grigor'eva, and V. K. Potapov, Khim. Vys. Energ. 18, 195 (1984).

${ }^{39}$ C. R. Silva and J. P. Reilley, J. Phys. Chem. A 101, 7934 (1997)

${ }^{40}$ L. Zhu and T. J. Cronin, Chem. Phys. Lett. 317, 227 (2000).

${ }^{41}$ V. S. Antonov, I. N. Knyazev, V. S. Letokhov, V. M. Matyuk, V. G. Movshev, and V. K. Potapov, Khim. Vys. Energ. 12, 476 (1978); V. S. Antonov and V. S. Letokhov, Appl. Phys. 24, 89 (1981); V. S. Antonov, Opt. Spektrosk. 52, 10 (1982).

${ }^{42}$ M. A. Grela and A. J. Colussi, J. Phys. Chem. 90, 434 (1986).

${ }^{43}$ J. A. Warren and E. R. Bernstein, J. Chem. Phys. 85, 2365 (1986).

${ }^{44}$ M. Koyanagi, R. J. Zwarich, and L. Goodman, J. Chem. Phys. 56, 3044 (1972).

${ }^{45}$ M. Berger and C. Steel, J. Am. Chem. Soc. 97, 4817 (1975).

${ }^{46}$ A. Inoue, M. Ushiyama, and N. Ebara, Chem. Phys. Lett. 117, 18 (1985); A. R. Rennert and C. Steel, ibid. 78, 36 (1981); G. A. Zalesskaya, D. L. Yakovlev, and E. G. Sambor, Opt. Spektrosk. 86, 205 (1999).
${ }^{47}$ Y. Hirata and E. C. Lim, Chem. Phys. Lett. 71, 167 (1980).

${ }^{48}$ A. P. Darmanyan and C. S. Foote, J. Phys. Chem. 97, 4573 (1993)

${ }^{49}$ A. P. Baronavski and J. C. Owrutsky, Chem. Phys. Lett. 333, 36 (2001).

${ }^{50}$ J. Dobkowksi, Z. R. Grabowski, J. Waluk, W. Kühne, W. Rettig, C. Rullière, W. Yang, J. Adamus, and J. Gebicki, Proc. Indian Acad. Sci., Math. Sci. 104, 143 (1992).

${ }^{51}$ S. Srivistava, E. Yourd, and J. P. Toscano, J. Am. Chem. Soc. 120, 6173 (1998).

${ }^{52}$ S. Anand, M. M. Zamari, G. Menkir, R. J. Levis, and H. B. Schlegel, J. Phys. Chem. A 108, 3162 (2004).

${ }^{53}$ H.-Q. Zhao, Y.-S. Cheung, C.-L. Liao, C.-X. Liao, C. Y. Ng, and W.-K. Li, J. Chem. Phys. 107, 7230 (1997).

${ }^{54}$ R. Srinivasan, V. A. Lobastov, C.-Y. Ruan, and A. H. Zewail, Helv. Chim. Acta 86, 1763 (2003).

${ }^{55}$ H. Ihee, B. M. Goodson, R. Srinivasan, V. A. Lobastov, and A. H. Zewail, J. Phys. Chem. A 106, 4087 (2002).

${ }^{56}$ M. Dantus, S. B. Kim, J. C. Williamson, and A. H. Zewail, J. Phys. Chem. 98, 2782 (1994); J. C. Williamson, J. Cao, H. Ihee, H. Frey, and A. H. Zewail, Nature (London) 386, 159 (1997).

${ }^{57}$ J. C. Williamson and A. H. Zewail, Chem. Phys. Lett. 209, 10 (1993).

${ }^{58}$ M. J. Frisch, G. W. Trucks, H. B. Schlegel et al., GAUSSIAN 98 (Gaussian Inc., Pittsburgh, PA, 1998).

${ }^{59}$ M. W. Schmidt, K. K. Baldridge, J. A. Boatz et al., J. Comput. Chem. 14, 1347 (1993).

${ }^{60}$ A. D. Becke, J. Chem. Phys. 98, 5648 (1993); R. H. Hertwig and W. Koch, Chem. Phys. Lett. 268, 345 (1997); P. J. Stephens, F. J. Devlin, C. F. Chabalowski, and M. J. Frisch, J. Phys. Chem. 98, 11623 (1994).

${ }^{61}$ J. M. Martell, H. Yu, and J. D. Goddard, Mol. Phys. 92, 497 (1997).

${ }^{62}$ H. Y. Afeefy, J. F. Liebman, and S. E. Stein, in NIST Chemistry WebBook, NIST Standard Reference Database Number 69, edited by P. J. Linstrom and W. G. Mallard (National Institute of Standards and Technology, Gaithersburg, MD, 2005).

${ }^{63}$ M. W. Schmidt and M. S. Gordon, Annu. Rev. Phys. Chem. 49, 233 (1998); B. O. Roos, in Methods in Computaional Molecular Physics, edited by G. H. F. Diercksen and S. Wilson (Reidel, Dordrecht, Netherlands, 1983), p. 161.

${ }^{64}$ H. Nakano, J. Chem. Phys. 99, 7983 (1993).

${ }^{65}$ E. W.-G. Diau, C. Kötting, and A. H. Zewail, ChemPhysChem 2, 273 (2001).

${ }^{66}$ Stereochemical Applications of Gas-Phase Electron Diffraction. Part A: The Electron Diffraction Technique, edited by I. Hargittai and M. Hargittai (VCH, New York, 1988).

${ }^{67}$ A. W. Ross, M. Fink, R. Hilderbrandt, J. Wang, and V. H. Smith, Jr., in International Tables for Crystallography, edited by A. J. C. Wilson and E. Prince (Kluwer, Boston, 1999), Vol. C, p. 262.

${ }^{68}$ V. S. Mastryukov and S. J. Cyvin, J. Mol. Struct. 29, 15 (1975); V. S. Mastryukov, E. L. Osina, L. V. Vilkov, and S. J. Cyvin, Zh. Strukt. Khim. 17, 80 (1976); E. L. Osina, V. S. Mastryukov, L. V. Vilkov, and S. J. Cyvin, ibid. 16, 1065 (1975).

${ }^{69}$ D. Shorokhov, S. T. Park, and A. H. Zewail, ChemPhysChem 6, 2228 (2005).

${ }^{70}$ M. M. Lin, D. Shorokhov, and A. H. Zewail, Chem. Phys. Lett. 420, 1 (2006).

${ }^{71}$ J. C. Williamson and A. H. Zewail, J. Phys. Chem. 98, 2766 (1994); J. S. Baskin and A. H. Zewail, ChemPhysChem 6, 2261 (2005).

${ }^{72}$ W. H. Press, S. A. Teukolsky, W. T. Vetterling, and B. P. Flannery, Numerical Recipes in C: The Art of Scientific Computing (Cambridge University Press, Cambridge, 1999).

${ }^{73}$ J. R. Taylor, An Introduction to Error Analysis. The Study of Uncertainties in Physical Measurements, 2nd ed. (University Science Books, Sausilito, 1997).

${ }^{74}$ R. J. Mawhorter, M. Fink, and B. T. Archer, J. Chem. Phys. 79, 170 (1983).

${ }^{75}$ K. A. Holbrook, M. J. Pilling, and S. H. Robertson, Unimolecular Reactions, 2nd ed. (Wiley, New York, 1996).

${ }^{76}$ J. G. Calvert and J. N. Pitts, Jr., Photochemistry (Wiley, New York, 1967); A. Horowitz and J. G. Calvert, J. Phys. Chem. 86, 3105 (1982); A. Horowitz, C. J. Kershner, and J. G. Calvert, ibid. 86, 3094 (1982); J. S. Yadav and J. D. Goddard, J. Chem. Phys. 84, 2682 (1986); B. F. Gherman, R. A. Friesner, T.-H. Wong, Z. Min, and R. Bersohn, ibid. 114, 6128 (2001); Y. Kurosaki and K. Yokoyama, Chem. Phys. Lett. 371, 568 (2003)

${ }^{77}$ R. G. W. Norrish, H. G. Crone, and O. D. Saltmarsh, J. Chem. Soc. 1456 
(1934); Y. Haas, Photochem. Photobiol. Sci. 3, 6 (2004).

${ }^{78}$ W.-K. Chen, J.-W. Ho, and P.-Y. Cheng, J. Phys. Chem. A 109, 6805 (2005); W.-K. Chen and P.-Y. Cheng, ibid. 109, 6818 (2005).

${ }^{79}$ A. B. Burrill, J. T. Zhou, and P. M. Johnson, J. Phys. Chem. A 107, 4601 (2003).
${ }^{80}$ D. G. Wilden and J. Comer, J. Phys. B 13, 627 (1980).

${ }^{81}$ P. Swiderek, M. Michaud, and L. Sanche, J. Chem. Phys. 105, 6724 (1996).

${ }^{82}$ E. Yamamoto, T. Yoshidome, T. Ogawa, and H. Kawazumi, J. Electron Spectrosc. Relat. Phenom. 63, 341 (1993). 